\title{
The genome sequence of Rickettsia prowazekif and the origin of mitochondria
}

\author{
Siv G. E. Andersson ${ }^{\star}$, Alireza Zomorodipour ${ }^{\star}$, Jan O. Andersson, Thomas Sicheritz-Pontén, \\ U. Cecilia M. Alsmark, Raf M. Podowski ${ }^{\star}$, A. Kristina Näslund ${ }^{\star}$, Ann-Sofie Eriksson ${ }^{\star}$, Herbert H. Winkler $\dagger$ \\ \& Charles G. Kurland*
}

* Department of Molecular Biology, University of Uppsala, Uppsala S-75124, Sweden

$\dagger$ Department of Microbiology and Immunology, University of South Alabama, Mobile, Alabama 36688, USA

\begin{abstract}
We describe here the complete genome sequence $(1,111,523$ base pairs) of the obligate intracellular parasite Rickettsia prowazekif, the causative agent of epidemic typhus. This genome contains 834 protein-coding genes. The functional profiles of these genes show similarities to those of mitochondrial genes: no genes required for anaerobic glycolysis are found in either $R$. prowazekii or mitochondrial genomes, but a complete set of genes encoding components of the tricarboxylic acid cycle and the respiratory-chain complex is found in $R$. prowazekii. In effect, ATP production in Rickettsia is the same as that in mitochondria. Many genes involved in the biosynthesis and regulation of biosynthesis of amino acids and nucleosides in free-living bacteria are absent from $R$. prowazekif and mitochondria. Such genes seem to have been replaced by homologues in the nuclear (host) genome. The $R$. prowazekii genome contains the highest proportion of non-coding DNA (24\%) detected so far in a microbial genome. Such non-coding sequences may be degraded remnants of 'neutralized' genes that await elimination from the genome. Phylogenetic analyses indicate that $R$. prowazekif is more closely related to mitochondria than is any other microbe studied so far.
\end{abstract}

The Rickettsia are $\alpha$-proteobacteria that multiply in eukaryotic cells only. $R$. prowazekii is the agent of epidemic, louse-borne typhus in humans. Three features of this endocellular parasite deserve our attention. First, R. prowazekii is estimated to have infected 20-30 million humans in the wake of the First World War and killed another few million following the Second World War (ref. 1). Because it is the descendent of free-living organisms ${ }^{2-4}$, its genome provides insight into adaptations to the obligate intracellular lifestyle, with probable practical value. Second, phylogenetic analyses based on sequences of ribosomal RNA and heat-shock proteins indicate that mitochondria may be derived from the $\alpha$ proteobacteria $^{5,6}$. Indeed, the closest extant relatives of the ancestor to mitochondria seem to be the Rickettsia ${ }^{7-10}$. That modern Rickettsia favour an intracellular lifestyle identifies these bacteria as the sort of organism that might have initiated the endosymbiotic scenario leading to modern mitochondria ${ }^{11}$. Finally, the genome of $R$. prowazekii is a small one, containing only $1,111,523$ base pairs (bp). Its phylogenetic placement and many other characteristics identify it as a descendant of bacteria with substantially larger genomes $^{2-4}$. Thus Rickettsia, like mitochondria, are good examples of highly derived genomes, the products of several types of reductive evolution.

The genome sequence of $R$. prowazekii indicates that these three features may be related. For example, prokaryotic genomes evolving within a cell dominated by a much larger, eukaryote genome and constrained by bottle-necked population dynamics will tend to lose genetic information ${ }^{12,13}$. Predictable sets of expendable genes will tend to disappear from the prokaryotic genome when they are made redundant by the activities of nuclear genes. Likewise, non-essential sequences and otherwise highly conserved gene clusters may be obliterated by deleterious mutations that are fixed in clonal parasite or organelle populations because they cannot be eliminated by selection. This process is ongoing in the Rickettsia genomes, as shown by the identification of sequences that have recently become pseudogenes. Also, a large fraction $(\sim 25 \%)$ of non-coding sequences in this genome may be gene remnants that have been degraded by mutation and have not yet been removed from the genome. Finally, transfer of genes from a mitochondrial ancestor to the nucleus of the host would both reduce the mitochondrial genome size and stabilize the symbiotic relationship. Phylogenetic reconstructions that identify genes in the Rickettsia genome as sister clades to eukaryotic homologues found in the nucleus or the organelle support this interpretation. Rickettsia and mitochondria probably share an $\alpha$-proteobacterial ancestor and a similar evolutionary history.

\section{General features of the genome}

The circular chromosome of $R$. prowazekii strain Madrid E has $1,111,523$ bp and an average $\mathrm{G}+\mathrm{C}$ content of $29.1 \%$ (Figs 1,2). The genome contains 834 complete open reading frames with an average length of $1,005 \mathrm{bp}$. Protein-coding genes represent $75.4 \%$ of the genome and $0.6 \%$ of the genome encodes stable RNA. We have assigned biological roles to $62.7 \%$ of the identified genes and pseudogenes; $12.5 \%$ of the identified genes match hypothetical coding sequences of unknown function and the remaining $24.8 \%$ represent unusual genes with no similarities to genes in other organisms (Table 1). Multivariate statistical analysis has shown that there is no major variation in codon-usage patterns among genes that are expressed in different amounts, indicating that codon-usage patterns in $R$. prowazekii may be dominated mainly by mutational forces ${ }^{14}$. G+C-content values at the three codon positions average $40.4,31.2$ and $18.6 \%$, and these values are similar at different positions in the genome. We classified the open reading frames with significant sequence-similarity scores to gene sequences in the public databases into functional categories (Table 1) that allow comparisons with the metabolic profiles of other bacterial genomes $^{15-23}$.

Non-coding DNA. The coding content of previously sequenced bacterial genomes is, on average, $91 \%$, ranging from $87 \%$ in Haemophilus influenzae to $94 \%$ in Aquifex aeolicum. In comparison, a large fraction of the $R$. prowazekii genome, 24\%, represents noncoding DNA (Fig. 3). A small fraction of this corresponds to 


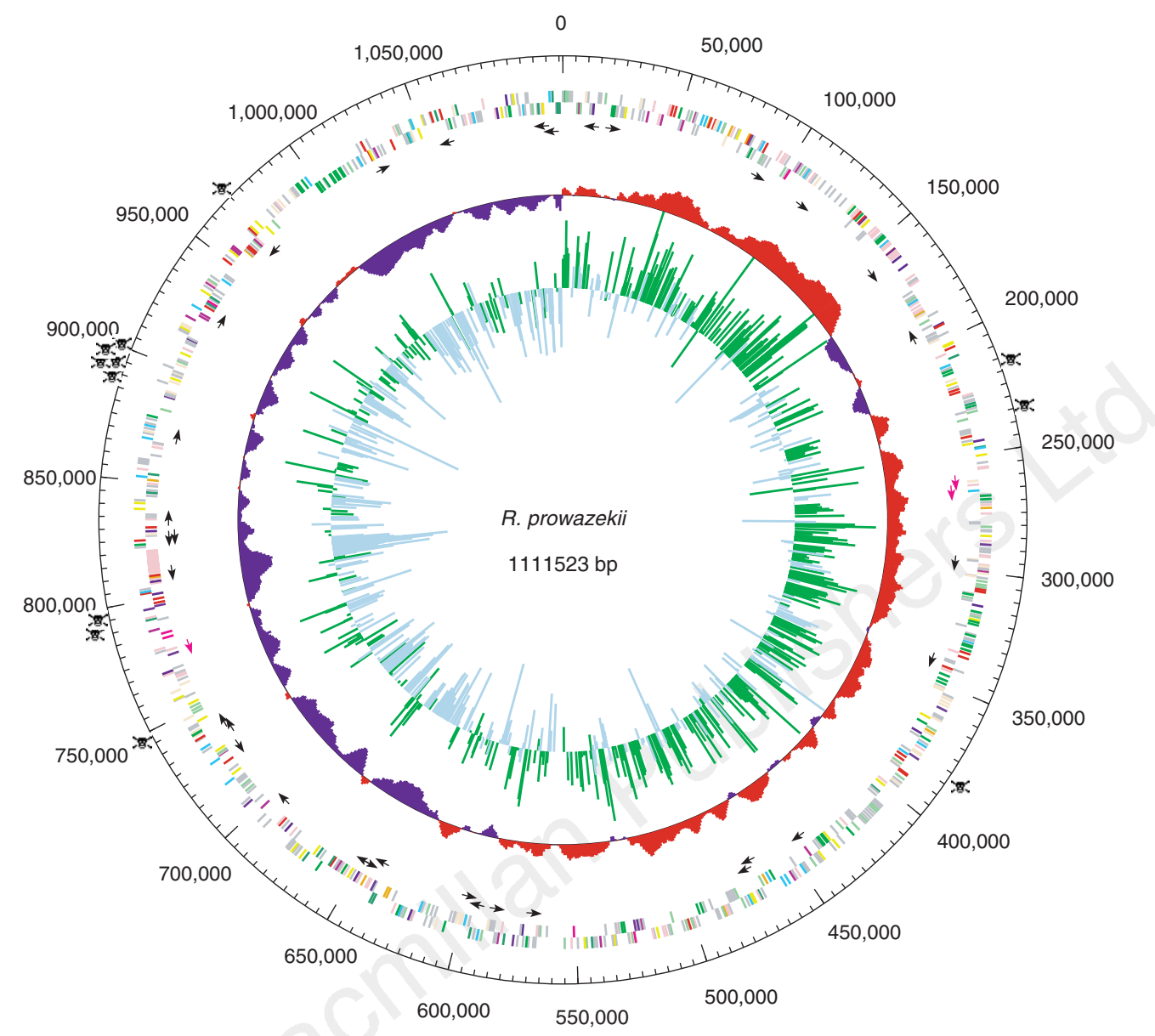

Figure 1 Overall structure of the $R$. prowazekii genome. The putative origin of replication is at $0 \mathrm{~kb}$. The outer scale indicates the coordinates (in base pairs). The positions of pseudogenes are highlighted with death's heads. The distribution of genes is shown on the first two rings within the scale. The location and direction of transcription of rRNA are shown by pink arrows and of tRNA genes by black arrows. The next circle in shows GC-skew values measured over all bases in the genome. Red and purple colours denote positive and negative signs, respec- tively. The window size was 10,000 nucleotides and the step size was 1,000 nucleotides. The central circles shows GC-skew values calculated for third positions in the codon only. GC-skew values were calculated separately for genes located on the outer strand (green) and on the inner strand (blue). To allow easier visual inspection, the signs of the values calculated for genes located on the inner strand have been reversed. pseudogenes $(0.9 \%$ of the genome) and less than $0.2 \%$ of the genome is accounted for by non-coding repeats. The remaining $22.9 \%$ contains no open reading frames of significant length and it has the low $\mathrm{G}+\mathrm{C}$ content (mean 23.7\%) that is characteristic of spacer sequences in the $R$. prowazekii genome ${ }^{14}$. A region of 30 kilobases $(\mathrm{kb})$ located at position $886-916 \mathrm{~kb}$ contains as much as $41.6 \%$ non-coding DNA and $11.5 \%$ pseudogenes. The non-coding DNA in this region has a small, but significantly higher, $\mathrm{G}+\mathrm{C}$ content (mean 27.3\%) than non-coding DNA in other areas of the genome (mean $23.7 \%)(P<0.001)$, indicating that it may correspond to inactivated genes that are being degraded by mutation (Fig. 3).

Origin of replication. The origin of replication has not been experimentally identified in the $R$. prowazekii genome, but we identified $d n a A$ at $\sim 750 \mathrm{~kb}$. However, the genes flanking the $d n a A$ gene differ from the conserved motifs found in Escherichia coli and Bacillus subtilis ( $r n p A-r p m H-d n a A-d n a N-r e c F-g y r B)$. In R. prowazekii, the genes rnpA and $r p m H$ are located in the vicinity of $d n a A$, but in the reverse orientation compared to the consensus motif, and $d n a N, \operatorname{recF}$ and $g y r B$ are located elsewhere.

The origin and end replication in microbial genomes are often associated with transitions in $\mathrm{GC}$ skew $(\mathrm{G}-\mathrm{C} / \mathrm{G}+\mathrm{C})$ values $^{24}$. In $R$. prowazekii we observe transitions in the GC skew values at around 0 and $500-600 \mathrm{~kb}$ (Fig. 1). There is a weak asymmetry in the distribution of genes in the two strands, such that the first half of the genome has a 1.6-fold higher gene density on one strand and the second half of the genome has a 1.6-fold higher gene density on the other strand. The shift in coding-strand bias correlates with the shift in GC-skew values. As most genes are transcribed in the direction of replication in microbial genomes, the origin of replication may correspond to the shift in GC-skew values at the position that we have chosen as the start point for numbering. Indeed, several short sequence stretches that are characteristic of $d n a A$-binding motifs are found in the intergenic region of genes RP001 and RP885 at $0 \mathrm{~kb}$, supporting this interpretation.

Stable RNA sequences and repeat elements. We identified 33 genes encoding transfer RNA, corresponding to 32 different isoacceptortRNA species. There is a single copy of each of the rRNA genes, with $r r s$ located more than $500 \mathrm{~kb}$ away from the $r r l-r r f$ gene cluster

Figure 2 Linear map of the R. prowazekii chromosome. The position and orientation of known genes are indicated by arrows. Coding regions are colourcoded according to their functional roles. The positions of tRNA genes are indicated (inverted triangle on stalk). For additional information, see http:// evolution.bmc.uu.se/ siv/gnomics/Rickettsia.html. 
(Fig. 1). Comparison of the sequences from ten different Rickettsia species indicates that the disruption of the rRNA gene operon preceded the divergence of the typhus group and spotted fever group Rickettisia (S.G.E.A. et al., unpublished observations). In addition, the genome contains a short sequence with similarity to a 213-nucleotide RNA molecule in Bradyrhizobium japonicum that may regulate transcription ${ }^{25}$.

There are unusually few repeat sequences in this genome. We identified four different types of repeat sequence: all of these are located in intergenic regions. There is a sequence of $80 \mathrm{bp}$ that is repeated seven times downstream of $r p m H$ and $r n p A$ in the $d n a A$ region. A repetitive sequence of $325 \mathrm{bp}$ is found at two intergenic regions that are more than $80 \mathrm{~kb}$ apart, downstream of the genes $k s g A$ and $r n h$, respectively. A 440-bp-long repetitive sequence has been identified at two intergenic sites, $140 \mathrm{~kb}$ apart; one of these sites is downstream of $\operatorname{rrf}$ and the others downstream of $p d h A$ and

Table 1 Asterisks indicate putative pseudogenes. Abbreviations of species names are: Bacteria: Acinetobacter calcoaceticus (B-Aca), Actonobacillus actinomycetemcomitans (B-Aac), Acyrthosiphon condii (B-Aco), Agrobacterium tumefaciens (B-Atu), Alcaligenes eutrophus (B-Aeu), Anabena sp. PCC7120 (B-Asp), Anabena variabilis (B-Ava), Anacystis nidulans (B-Ani), Azorhizobium caulinodans (B-Aca), Azosprillium brasiliense (B-Abr), Azotobacter vinelandii (B-Avi), Bacillus caldotenax (B-Bca), Bacillus stereothermophilus (B-Bst), Bacillus subtilis (B-Bsu), Bartonella bacilliformis (B-Bba), Bartonella henselae (B-Bhe), Bordetella pertussis (B-Bpe), Borrelia burgdorferi (B-Bbu), Bradyrhizobium japonicum (B-Bja), Brucella abortus (B-Bab), Brucella ovis (B-Bov), Caulobacter crescentus (B-Ccr), Chlamydia trachomatis (B-Ctr), Chloroflexus aurantiacus (B-Cau), Chromatum viosum (B-Cvi), citrus-greening-disease-associated bacterium (B-Cgr), Clostridium acetobutylicum (B-Cac), Clostridium pasteurianum (B-Cpa), Clostridium thermosaccharolyticum (B-Cts), Coxiella burnetii (B-Cbu), Erwinia chrysanthemi (B-Ech), Escherichia coli (B-Eco), Haemophilus influenzae (B-Hin), Helicobacter pylori (B-Hpy), Klebsiella pneumoniae (B-Kpn), Legionella pneumophila (B-Lpn), Leucothrix mucor (B-Lmu), Liberobacter africanum (B-Laf), Methylobacterium extorquens (B-Mex), Micrococcus luteus (Mlu), Moraxella catarrhalis (Mca), Mycobacterium leprae (M/e), Mycobacterium smegmatis (B-Msm), Mycobacterium tuberculosis (B-Mtu), Mycoplasma capricolum (B-Mca), Mycoplasma genitalium (B-Mge), Mycoplasma pneumoniae (B-Mpn), Paracoccus denitrificans (B-Pde), Pasteurella haemolytica (B-Pha), Plectonema boryanum (B-Pbo), Proteus mirabilis (B-Pmi), Proteus vulgaris (BPvu), Pseudomonas aeruginosa (B-Pae), Pseudomonas fluorescens (B-Pf), Pseudomonas putida (B-Ppu), Pseudomonas syringae (B-Psy), Rhizobium meliloti (B-Rme), Rhizobium sp. NGR234 (B-Rsp), Rhodobacter capsulatus (B-Rca), Rhodobacter sphaeroides (B-Rsp), Rhodobacter sulfidophilus (B-Rsu), Rhodopseudomonas blastica (B-Rb/), Rhodospirillum rubrum (B-Rru), Rickettsia japonicum (B-Rja), Rickettsia rickettsii (B-Rri), Rickettsia typhi (B-Rty), Salmonella typhi (B-Sti), Salmonella typhimurium (B-Sty), Shigella flexneri (B-Sf), Sprioplasma citri (B-Sci), Staphylococcus aureus (B-Sau), Staphyloccus carnosus (B-Sca), Streptococcus pneumoniae (B-Spn), Streptomyces clavuligerus (B-Scl), Streptomyces coelicor (B-Sco), Synechocystis PCC 6803 (B-Syn), Thermus aquaticus (B-Taq), Thermus thermophilus (B-Tth), Thiobacillus cuprinus (B-TCU), Treponema hyodysenteriae (B-Thy), Vibrio alginolytics (B-Val), Vibrio cholera (B-Vch), Vibrio parahaemolyticus (B-Vpa), Vibrio proteolyticus (B-Vpr), Wolbachia sp. (B-Wsp), Yersinia entercolitica (B-Yen), Zooglea ramigera (B-Zra), Zymomonas mobilis (B-Zmo). Archaea: Methanococcus jannaschii (A-Mja), Sulfulobus acidocaldarius (A-Sac). Eukaryotes: Apis mellifera (E-Ame), Arabidopsis thaliana (E-Ath), Atratylodes japonica (E-Aja), Bos taurus (E-Bta), Candida albicans (E-Cal), Caenorhabidits elegans (E-Cel), Dictylostelium discoideum (E-Ddi), Flaveria trinervia (E-Ftr), Giardia theta (E-Gth), Glycine max (EGma), Haematobia irritans (E-Hir), Homo sapiens (E-Hsa), Marchantia polymorpha (E-Mpa), Mus musculum (E-Mmu), Prototheca wickerhamii (E-Pwi), Petunia hybrida (E-Phy), Pisum sativum (E-Psa), Porphyra purpurea (E-Ppu), Odontella sinensis (E-Osi), Reclinomonas americana (E-Ram), Rattus novergicus (E-Rno), Rhizopus oryzae (E-Ror), Saccharomyces cerevisiae (E-Sce), Schizosaccharomyces pombe (E-Spo), Solanum tuberosum (E-Stu), Spinacia oleracea (E-Sol). $p d h B$. Finally, two similar sequences of $730 \mathrm{bp}$ are located immediately next to each other at $850 \mathrm{~kb}$.

Paralogous families. We have identified 54 paralogous gene families comprising 147 gene products. Of these, 125 have an assigned function. Most paralogues encode proteins with transport functions, such as the $\mathrm{ABC}$ transporters, the proline/betaine transporters and the ATP/ADP transporters. Five paralogous genes located next to each other at $115 \mathrm{~kb}$ encode putative integral membrane proteins with unknown functions.

\section{Biosynthetic pathways}

A striking feature of the $R$. prowazekii genome is the small proportion of biosynthetic genes compared with free-living proteobacterial relatives (such as Haemophilus influenzae, Helicobacter pylori and E. coli $)^{15,19,20}$. This scarcity of biosynthetic functions is also seen in diverse endocellular and epicellular parasites ${ }^{16-18,23}$. This scarcity of biosynthetic functions is also seen in diverse endocellular and epicellular parasites ${ }^{16-18,23}$.

Amino-acid metabolism. As many as 43 and 69 genes required for amino-acid biosynthesis are found in Helicobacter pylori and Haemophilus influenzae, respectively. In contrast, Mycoplasma genitalium and Borrelia burgdorferi contain only glyA, which encodes serine hydroxymethyltransferase. This gene is also found in $R$. prowazekii (Table 1). Serine hydroxymethyltransferase catalyses the conversion of serine and tetrahydrofolate into glycine and methylenetetrahydrofolate, respectively. A role in tetrahydrofolate metabolism may account for the ubiquity of $g l y A$ in bacteria.

Seven genes normally associated with lysine biosynthesis (lys $C$, asd, $\operatorname{dap} A, d a p B, d a p D, d a p E$ and $d a p F)$ are also present in $R$. prowazekii. The biosynthetic pathways leading to lysine, methionine and threonine share the first two of these (lys $C$ and $a s d)$. However, none of the downstream genes for threonine biosynthesis are found in $R$. prowazekii. Likewise, the lysine pathway is incomplete, and lys $A$, which encodes the enzyme that converts meso-diaminopimelate to lysine, is missing. The likely role of the upstream genes of this pathway in $R$. prowazekii is the biosynthesis of diaminopimelate, an essential envelope component. We have therefore classified these genes as 'cell-envelope' genes (Table 1).

We have identified other genes that are superficially involved in the metabolism of amino acids, but which apparently function in deamination pathways that divert amino acids into the tricarboxylic acid (TCA) cycle. For example, there is aatA, encoding aspartate aminotransferase, which catalyses the degradation of aspartate to oxaloacetate and glutamate. $t d c B$ encodes threonine deaminase, which converts threonine into $\alpha$-ketobutyrate. Another gene $(i l v E)$ encodes branched-chain-amino-acid aminotransferase, which converts leucine, isoleucine or valine into glutamate. $p c c A$ and $p c c B$ encode propionyl-CoA carboxylase, which converts propionyl$\mathrm{CoA}$, an intermediate in the breakdown of methionine, valine and isoleucine, into succinyl-CoA. The $p c c A$ and $p c c B$ gene products show greatest similarity to the eukaryotic proteins that are located in the mitochondrial matrix.

Nucleotide biosynthesis. No genes required for the de novo syntheses of nucleosides have been found in the $R$. prowazekii genome. However, four genes required for the conversion of nucleoside monophosphates into nucleoside diphosphates ( $a d k, g m k, c m k$ and $p y r H)$ are present. There are also two genes encoding ribonucleotide reductase, which converts ribonucleoside diphosphates into deoxyribonucleoside diphosphates. Nucleoside diphosphate kinase (encoded by $n d k$ ), which converts NDPs and dNDPs to NTPs and dNTPs, is also present in $R$. prowazekii. Finally, there is a complete set of genes for the conversion of dCTP and dUTP into TTP, including thyA, which codes for thymidylate synthase. Thus, the $R$. prowazekii genome encodes all of the enzymes required for the interconversion of nucleoside monophosphates into all of the other required nucleotides. The nucleoside monophosphates are probably imported from the eukaryotic host. 


\section{Energy metabolism}

Early in its infectious cycle, $R$. prowazekii uses the ATP of the host with the help of membrane-bound ATP/ADP translocases. However, $R$. prowazekii is also capable of generating ATP, which may compensate for the gradual depletion of cytosolic ATP later in the infection. $R$. prowazekii's repertoire of genes involved in ATP production and transport include determinants for the TCA cycle, the respiratory-chain complexes, the ATP-synthase complexes and the ATP/ADP translocases (Table 1). Genes to support anaerobic glycolysis are absent.

Pyruvate dehydrogenase. Pyruvate is imported into mitochondria directly from the cytoplasm and converted into acetyl-CoA by pyruvate dehydrogenase. The genes encoding three components (E1-E3) of the pyruvate dehydrogenase complex are found in $R$. prowazekii, indicating that it too uses cytosolic pyruvate. Pyruvate dehydrogenase (E1) consists of two subunits $(\alpha$ and $\beta$ ) in $R$. prowazekii, mitochondria and Gram-positive bacteria; the corresponding genes are clustered in the genome. In contrast, proteobacteria such as E. coli, Haemophilus influenzae and Helicobacter pylori have a single subunit for the E1 component and these have little similarity to the $\alpha$ and $\beta$ subunits of the $\mathrm{E} 1$ component in $R$. prowazekii and mitochondria (data not shown).

Two paralagous genes code for the dihydrolipoamide dehydrogenase (E3) in $R$. prowazekii. One of these most resembles mitochondrial homologues, whereas the other is most similar to bacterial homologues (data not shown). The presence of several paralogous gene families for pyruvate dehydrogenases complicates attempts to reconstruct a genome phylogeny based on these genes. ATP production. Genes encoding all enzymes in the TCA cycle are found in $R$. prowazekii. Proton translocation is mediated by NADH dehydrogenase (complex I), cytochrome reductase (complex III) and cytochrome oxidase (complex IV). Several clusters of genes code for components of the NADH dehydrogenase complex. Seven of these genes (nuoJKLM and nuoGHO) are located near to each other, but the order of genes is inverted relative to the order of this cluster in E. coli. An additional set of five genes is grouped in the order $n u \circ A B C D E$, but the single genes $n u o F$ and $n u o N$ are distant from both of these clusters. Several proteins in the cytochrome $b c_{1}$ reductase complex, such as ubiquinol-cytochrome $c$ reductase (encoded by pet $A$ ), cytochrome $b$ (encoded by $c y t b$ ) and cytochrome $c_{1}$ (encoded by $f b h C$ ), are present, as are several subunits of the cytochrome oxidase complex.

The ATP-synthesizing complex is composed of the ATP synthase $\mathrm{F}_{1}$ component (comprising five polypeptides, $\alpha, \beta, \gamma, \epsilon$ and $\delta$ ) and the $\mathrm{F}_{\mathrm{o}}$ component, a hydrophobic segment that spans the inner mitochondrial membrane. The genes encoding these components are normally clustered in one of the most highly conserved operon structures in microbial genomes. In $R$. prowazekii, however, the ATP-synthase genes encoding the $\alpha, \beta, \gamma, \delta$ and $\epsilon$ subunits of the $F_{1}$ complex (atpH, atpA, atpG, atpD and atpC) are clustered in the common order, but atpB, atpE and atpF, encoding the $\mathrm{A}, \mathrm{B}$ and $\mathrm{C}$ chains of the $\mathrm{F}_{\mathrm{o}}$ complex, are split from this cluster.

\section{Replication, repair and recombination}

$R$. prowazekii has a smaller set of genes involved in DNA replication than do free-living bacteria such as E. coli, Haemophilus influenzae and Heliocobacter pyrlori. Four genes have been identified that code for the core structure of DNA polymerase III, which includes the $\alpha$ $(d n a E), \epsilon(d n a Q), \beta(d n a N), \gamma$ and $\theta(d n a X)$ subunits. Extra subunits present in the $E$. coli DNA polymerase III are missing from $R$. prowazekii, as well as from M. genitalium and B. burgdorferi.

Genes encoding DNA-repair mechanisms are similar in the small genomes of the parasites $R$. prowazekii, M. genitalium and $B$. burgdorferi. Thus, genes involved in the repair of ultravioletinduced DNA damage (uvrABCD) have been identified in all three genomes. In $R$. prowazekii, DNA-excision repair probably occurs by a pathway involving endonuclease III, polI and DNA ligase, as in B. burgdorferi.

The $R$. prowazekii genome has a limited capacity for mismatch repair. The DNA-mismatch-repair enzymes encoded by mutL and mutS are present, but $m u t H$ and $m u t Y$ are not. There is a complete lack of $m u t$ genes in M. genitalium, but mutL and mutHLY have been identified in B. burgdorferi and Chlamydia trachomatis. The transcriptionrepair coupling factor (encoded by $m f D$ ) is found in $R$. prowazekii, B. burgdorferi and C. trachomatis but not in M. genitalium.

The $R$. prowazekii genome contains several genes involved in homologous recombination, such as $r e c A$, recF, recJ, recN and $r e c R$. A similar set of genes has been found in A. aeolicus ${ }^{21}$. The rec genes

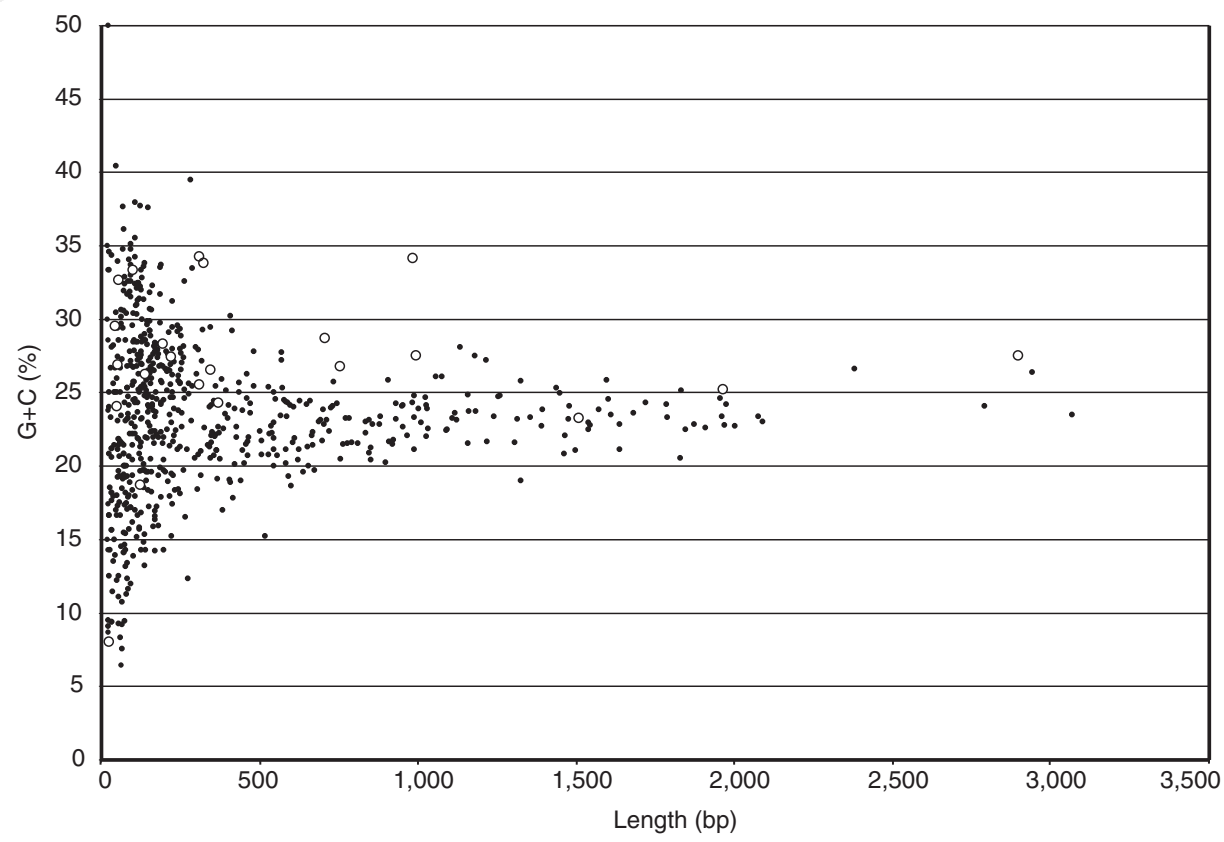

Figure $3 \mathrm{G}+\mathrm{C}$ content in intergenic regions longer than $20 \mathrm{bp}$ in the $R$. prowazekii genome. The empty circles correspond to spacer sequences located at 886 to 
are scattered in the other small genomes of parasites. The $\operatorname{RecBCD}$ complex is missing in R. prowazekii, M. genitalium and Helicobacter pylori but it has been identified in B. burgdorferi.

\section{Transcription and translation}

R. prowazekii has three subunits $\left(\alpha, \beta\right.$ and $\left.\beta^{\prime}\right)$ of the core RNA polymerase, as well as $\sigma^{70}$ and one alternative $\sigma$ factor, $\sigma^{32}$, which controls transcription of the genes encoding heat-shock proteins in E. coli. Genes involved in transcription elongation and termination, $n u s A, n u s B$, nus $G$, gre $A$ and $r h o$, are also present. The gene encoding $\sigma^{32}$ is absent in most other small genomes, such as those of B. burgdorferi, Helicobacter pylori, M. genitalium and C. trachomatis, although genes for heat-shock proteins are present.

An unusually large number of genes involved in RNA degradation are found in R. prowazekii. Of these, only four appear to be common to the bacterial genomes analysed so far (those encoding polyribonucleotide nucleotidyltransferase and ribonucleases HII, III and P). Four more ribonucleases (D, E, HI and $\mathrm{PH}$ ) are present in $R$. prowazekii, but in none of the other small parasites.

Of the 33 identified tRNA genes, which code for 32 different tRNA isoacceptor species, two code for tRNA ${ }^{\text {Phe }}$. There are two tRNA species for most of the amino acids that are encoded by fourcodon boxes; the exceptions are the four-codon boxes for proline and valine, for which we have identified only one isoacceptor-tRNA species, with $\mathrm{U}$ in the first anticodon position. selC, which codes for $\mathrm{tRNA}^{\mathrm{Sec}}$, and selABD are missing. $R$. prowazekii has a set of genes coding for tRNA modifications (tgt, queA, trmD, truA, truB and miaA) which resembles that of Helicobacter pylori, $C$. trachomatis and B. burgdorferi; $M$. genitalium has only trmD and truA.

In $R$. prowazekii, 21 genes encode 18 of the 20 aminoacyl-tRNA synthetases normally required for protein synthesis. There are two genes $(g l t X)$ encoding glutamyl-tRNA synthetase. As seen in several bacterial genomes ${ }^{25}$, the gene coding for glutaminyl-tRNA synthetase, $g \ln S$, is missing. Three genes encoding subunits of the glutamyl-tRNA amidotransferase are present, indicating that a glutamyl-tRNA charged with glutamic acid may be transamidated to generate Gln-tRNA. The gene coding for asparaginyl-tRNA synthetase, asnS, is also missing from the $R$. prowazekii genome as well as from Helicobacter pylori, C. trachomatis and A. aeolicus ${ }^{26}$. A transamidation process to form Asn-tRNA ${ }^{\text {Asn }}$ from Asp-tRNA ${ }^{\text {Asn }}$ has been proposed for the archaeon Haloferax volcanii ${ }^{27}$ and this reaction may also occur in $R$. prowazekii. The valyl-tRNA synthetase is $38.3 \%$ identical to its homologue in Methanococcus jannaschii, but only $27.6 \%$ identical to its most similar homologue in bacteria, which is found in Bacillus stearothermophilus, possibly indicating a horizontal transfer event. The lysyl-tRNA synthetase (encoded by $l y s S$ ) in $R$. prowazekii is a class I enzyme with no resemblance to the conventional class II lysyl-tRNA synthetases. Class I type of lysyltRNA synthetases have been observed previously in only B. burgdorferi, Pyrococcus woesii, Methanococcus jannaschii and a few other methanogens ${ }^{26}$.

\section{Regulatory systems}

As in other genomes of small parasites, $R$. prowazekii has a reduced set of regulatory genes. There are a few members of two-component regulatory systems, such as the proteins encoded by $\operatorname{bar} A$, env $Z$, $n \operatorname{tr} Y, n \operatorname{tr} X, o m p R$ and phoR. spoT, which is involved in the stringent response, has been identified in B. burgdorferi, Helicobacter pylori and M. genitalium. Only remnants of genes coding for aminoterminal fragments of proteins similar to those encoded by spoTand relA are identifiable in $R$. prowazekii. No fragments of spoTencoding the carboxy-terminal segments of the protein have been identified in the genome.

\section{Cell division and protein secretion}

Proteins involved in detoxification, such as superoxide dismutase, and those involved in thiophen and furan oxidation are present in $R$. prowazekii. Two genes encoding haemolysins have also been identified, and an $R$. typhi homologue of tlyC exhibits haemolytic activities when expressed in E. coli (S. Radulovic, J. M. Troyer, B. Noden, S.G.E.A. and A. Azad, unpublished observations).

The data indicate that the basic mechanisms of cell division and secretion in $R$. prowazekii are similar to those in free-living proteobacteria. There is a common set of bacterial chaperones (encoded by dnaK, dnaJ, hslU, hslV, groEL, groEL, groES and $h t p G)$ and genes involved in the secA-dependent secretory system (secABDEFGY, ffH and $f t s Y)$. R. prowazekii has a significantly larger set of genes involved in peptide secretion than does M. genitalium.

\section{Membrane-protein analysis}

Many studies of $R$. prowazekii have focused on outer-surface membrane proteins because of their potential importance in bacterial detection and vaccination. The superficial lipopolysaccharide (LPS) molecule is important in the pathogenesis of $R$. prowazekii. LPS consists of a polysaccharide that is covalently linked to lipid A, the biosynthesis of which is catalysed by products of $l p x A B C D$, all of which are present in the $R$. prowazekii genome. These genes are clustered in $E$. coli, but $l p x A$ and $l p x D$ are separate from $l p x B$ and $l p x C$ in $R$. prowazekii. Three genes involved in the biosynthesis of the 3-deoxy-D-manno-octulosonic acid (KDO) residues reside in the $R$. prowazekii genome ( $k d s A, k d s B$ and $k d t A)$. Only one gene $(r f a J)$ with a putative function in outer-core biosynthesis has been identified.

We have identified a set of genes involved in the biosynthesis of murein and diaminopimelate and a set involved in the biosynthesis of fatty acids. These includes: $f a b D$, which is involved in the last step of the initiation phase of fatty-acid biosynthesis; four genes involved in the elongation cycle of fatty-acid biosynthesis ( $f a b F G H I$ ); and three genes involved in the first three steps of the synthesis of polar head groups ( $c d s A, p s s A$ and $p g s A)$. Finally, post-translational processing and addition of lipids to an $\mathrm{N}$-terminal cysteine require the gene products prolipoprotein diacylglycerol transferase $(\operatorname{lgt})$, prolipoprotein signal peptidase $(l s p A)$ and apolipoprotein:phosholipid $N$-acyl transferase $(\ln t)$. These are found in the genome with several genes involved in the degradation of fatty acids, such as fadA which encodes the 3-ketoacyl-CoA thiolase.

\section{Virulence}

The R. prowazekii genome contains several homologues of the VirB gene operon found in Agrobacterium tumefaciens. This gene family encodes proteins that direct the export of the T-DNA-protein complex across the bacterial envelope to the plant nuclei ${ }^{28} . R$. prowazekii has two homologues of VirB4 and one homologue each of VirB8, VirB9, VirB10, VirB11 and VirD4. The latter five genes are clustered with the gene $\operatorname{trbG}$, which is involved in conjugation in Agrobactrium tumefaciens. Homologues of the single-stranded DNA-binding proteins VirD2 and VirE2 are missing. In Agrobacterium tumefaciens, these proteins are bound to the transferred T-DNA, indicating different functions for the homologues of the VirB genes in $R$. prowazekii. Indeed, VirB proteins are homologous to components of the $E$. coli transport system for plasmids, as well as to components of the Pt1 transport machinery in Bordetella pertussis, which exports pertussis toxin ${ }^{28}$. A set of genes coding for VirB4 and several other VirB proteins has been identified in the cag pathogenicity island of Helicobacter pylori. In this species, the VirB proteins facilitate export of a factor that induces interleukin-8 secretion in gastric epithelial cells ${ }^{28}$. Thus, $R$. prowazekii may encode components of a transport system for both conjugal DNA transfer and protein export.

The virulence of Staphylococcus aureus has been correlated with the production of capsular polysaccharides in phagocytic assays and mouse lethality assays ${ }^{29,30}$. A cluster of ten capsule genes $($ capA-M) is involved in capsule biosynthesis in $S$. aureus strain $\mathrm{M}^{31}$. We have identified three $R$. prowazekii genes with sequence similarities to $S$. aureus cap genes. Two of these (capD and capM) are separated by ten 
genes, most of which are unknown genes or genes involved in the biosynthesis of LPS or techoic acid. Thus, $R$. prowazekii may produce components of a microcapsular layer that is involved in virulence.

\section{Reductive evolution}

Genome sequences of organisms enjoying an endosymbiotic lifestyle are at risk. The activities of homologous nuclear genes may render genes of the endosymbiont expendable and as a consequence they become vulnerable to obliteration by mutation. Good candidates for such purged genes in Rickettsia and mitochondria are genes required for amino-acid biosynthesis, nucleoside biosynthesis and anaerobic glycolyis. These and other genes would have been deleted when an ancestral genome first lived in a nucleated cell. Once genes essential to a free-living mode are lost, the endosymbiont becomes an obligate resident of its host.

Likewise, small, bottle-necked populations of bacteria infecting a eukaryotic cell will tend to accumulate deleterious mutations because selection cannot remove them from such clonal populations $^{13}$. The accumulation of such harmful but non-lethal mutations is referred to as 'Muller's ratchet' ${ }^{32}$ or 'near-neutral evolution ${ }^{33,34}$. The consequence of accumulation of these mutations will be the inactivation and eventual deletion of non-essential genes.

The first mutation that inactivates an expendable gene is likely to initiate a sequence of events in which subsequent mutations freely transform it, by degrees, from a pseudogene, to unrecognizable sequence, to small fragments, to extinction. In this sequence, mutations are released from amino-acid-coding constraints. Thus nucleotide substitutions will reflect the mutation bias of the genome. This bias can be estimated roughly by frequencies of third-position bases in the codons. For $R$. prowazekii, the bias of the third-position bases is $18 \% \mathrm{G}+\mathrm{C}$ rather than the $29 \% \mathrm{G}+\mathrm{C}$ average for the genome. So, as sequences age in $R$. prowazekii, their composition should gradually approach the low $\mathrm{G}+\mathrm{C}$ content of third codon positions. Nearly one-quarter of the $R$. prowazekii genome is composed of non-coding sequences, with a $\mathrm{G}+\mathrm{C}$ content lower than that of coding sequences $(25 \% \mathrm{G}+\mathrm{C}$ compared to $30 \%$; $P<0.001)$. Thus, much of the non-coding sequence may be remnants of coding sequences that are in the process of being eliminated from the genome.

The gene encoding $S$-adenosylmethionine synthetase (metK), which catalyses the biosynthesis of $S$-adenosylmethionine (SAM), illustrates the initiation of this process. The metK sequence in the strain of $R$. prowazekii studied here has a termination codon within a region of the gene that is otherwise highly conserved among

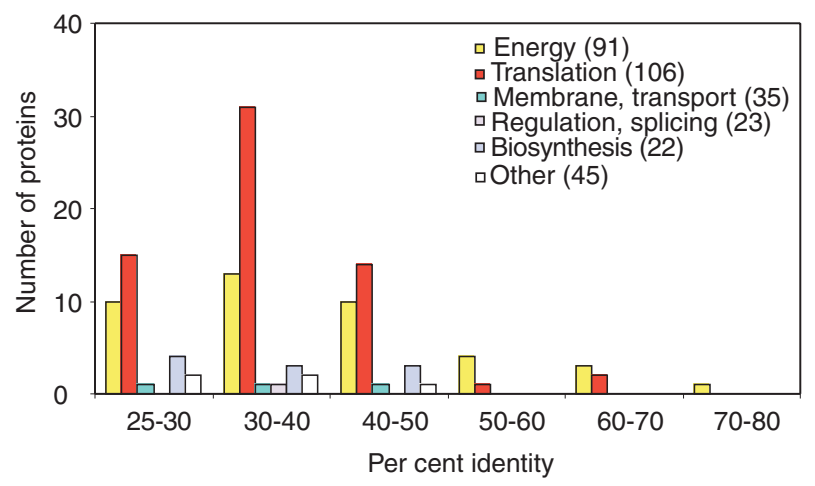

Figure 4 Histogram representation of the similarity of predicted $R$. prowazekii proteins to yeast proteins targeted to the mitochondria. Only protein pairs with per cent identity values greater than $25 \%$ are shown. Numbers in parentheses represent the total number of yeast mitochondrial proteins within each category. The yeast mitochondrial protein sequences have been taken from http:// www.proteome.com. bacterial species ${ }^{35}$. However, a closely related strain does not have the termination codon. Many other defects, such as termination codons, insertions, and a preponderance of small deletions, have also been observed in the metK genes in several members of the spotted fever group Rickettsia (J.O.A. and S.G.E.A., unpublished observations). This random distribution of lethal mutations among some $m e t K$ alleles from different Rickettsia species indicates that the gene may have just entered the extinction process. This distribution, and the identification of 11 more pseudogenes for carboxypeptidase $(y p w A)$, penicillin-binding protein $(p b p C)$, succinyl CoA-transferase $(s c o B)$, transposase (tra3), resolvase ( $p$ in $)$, conjugative transfer protein $(\operatorname{tax} B)$, a hypothetical protein $(y f c 1)$ and four different fragmented open reading frames for (p)ppGpp 3 '-pyrophosphohydrolase, indicates that the $R$. prowazekii genome continues to eliminate genes.

Genome sequences can be purged by a more abrupt mechanism. This consists of intrachromasomal recombination at duplicated sequences, which can result in the deletion of intervening sequences, the loss of a sequence duplication and the rearrangement of flanking

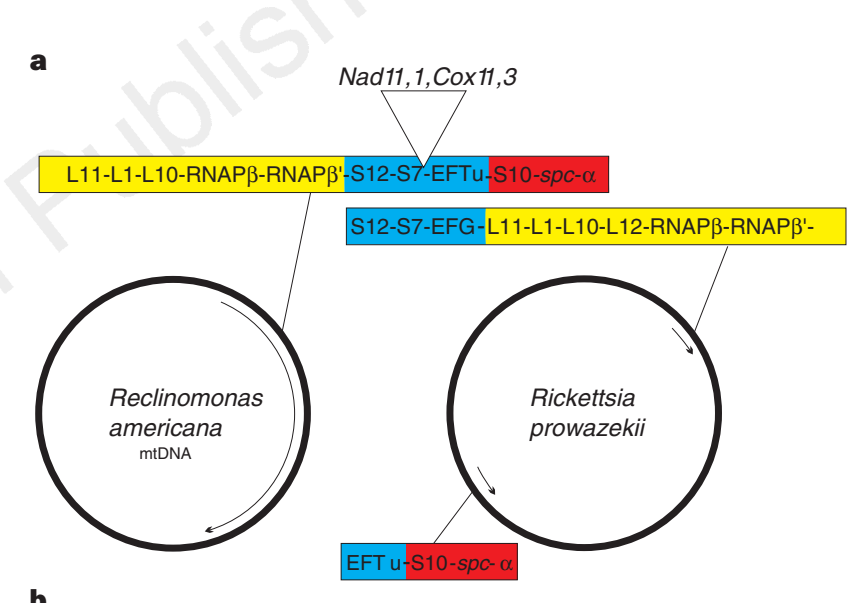

b

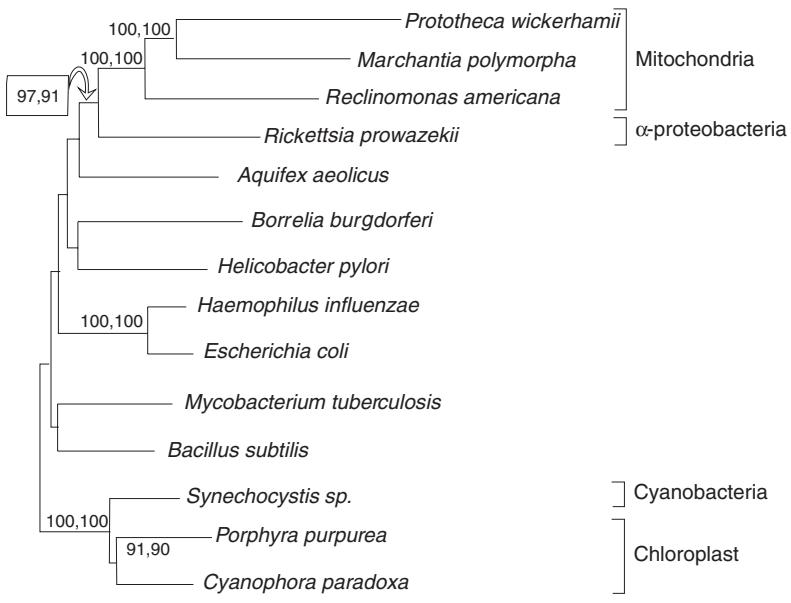

Figure 5 The organization and phylogenetic relationships of gene encoding ribosomal protein from $R$. prowazekii and the mitochondrial genome of Reclinomonas americana. a, The organization of ribosomal-protein genes. The $S 10, s p c$ and $\alpha$-operons are organized similarly in these two genomes, except that several ribosomal-protein genes ${ }^{38}$ have been deleted from the mitochondrial genome of Reclinomonas americana. b. The phylogenetic relationships of mitochondria and bacteria were derived from the combined amino-acid sequences of ribosomal proteins S2, S3, S7, S10, S11, S12, S13, S14, S19, L5, L6 and L16. Neighbour-joining and maximum-parsimony methods gave identical topologies. Branch lengths are proportional to those reconstructed by using the neighbour-joining method. Values at nodes are bootstrap values indicating the degree of support for individual clusters under each method (neighbour-joining, maximum parsimony). Only bootstrap values $>90 \%$ are shown. 
sequences. Such a mechanism will account for the presence in $R$. prowazekii of one, unlinked copy of $r r s$ and $r r l$, both of which are surrounded by new flanking sequences ${ }^{36}$. Likewise, $R$. prowazekii has one tuf gene and one fus gene in atypical clusters that seem to have been created by intrachromosomal recombination between the two tuf genes that are normally found in Gram-negative bacteria ${ }^{37}$. Indeed, rearranged gene operon structures encoding ribosomal proteins are characteristic of all members of the genus Rickettsia (H. Amiri, C.A. and S.G.E.A., unpublished observations).

Conserved operons that are found in free-living bacteria are often dispersed throughout the Rickettsia genome (see above). The $R$. prowazekii genome contains an unusually small fraction of repeat sequences $(<10 \%$ of that observed in free-living bacteria). We suggest that the repeat sequences found in the ancestor to the Rickettsia have been 'consumed' by the intrachromosomal-recombination mechanism that generated some of the deletions and rearrangements seen in $R$. prowazekii. Such intrachromosomal recombinants arise at a substantial rate in bacteria growing in culture, but here they are eliminated from the populations by selection. That such remnants of intrachromosomal recombination are retained in $R$. prowazekii indicates that purifying selection has been attenuated in this organism.

\section{Mitochondrial affinities}

The reduction in genome size in mitochondria and Rickettsia is likely to have occurred independently in the two lineages. Most of

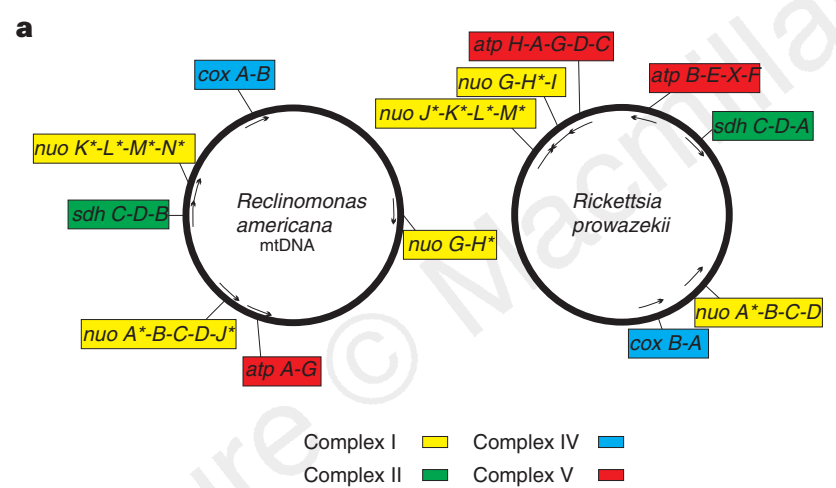

b

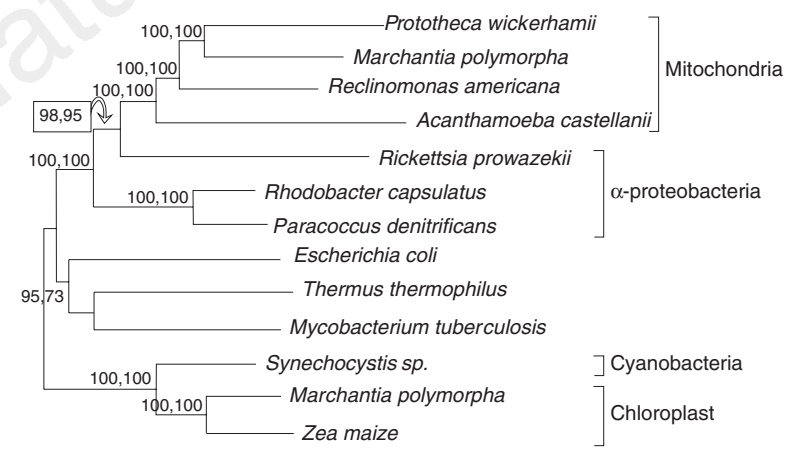

Figure 6 The organization and phylogenetic relationships of genes involved in ATP synthesis from $R$. prowazekii and the mitochondrial genome of Reclinomonas americana. a, The organization of bioenergetic genes. $\mathbf{b}$, The phylogenetic relationships of mitochondria and bacteria were derived from the combined amino-acid sequences of NADH dehydrogenase I chains $\mathrm{A}, \mathrm{J}, \mathrm{K}, \mathrm{L}, \mathrm{M}$ and $\mathrm{N}$, which are encoded by the genes nuoA, J, K, L, M, N. These genes are highlighted by asterisks in a. Neighbour-joining and maximum-parsimony methods gave identical topologies. Branch lengths are proportional to those reconstructed using the neighbour-joining method. Values at nodes are bootstrap values indicating the degree of support for individual clusters under each method (neighbour-joining, maximum parsimony). Only bootstrap values $>90 \%$ are shown. the genes supporting mitochondrial activities are nuclear. Many of the 300 proteins encoded in the nucleus of the yeast Saccharomyces cerevisiae but destined for service within the mitochondrion are close homologues of their counterparts in $R$. prowazekii. Nearly one-quarter of these proteins are required for bioenergetic processes and another one-third of them are required for the expression of the genes encoded in the mitochondrial genome. In total, more than 150 nucleus-encoded mitochondrial proteins share significant sequence homology with $R$. prowazekii proteins (Fig. 4).

Another group of 58 nucleus-encoded mitochondrial proteins represents components of the mitochondrial transport machinery and regulatory system (Fig. 4). These include proteins found in the mitochondrial outer membrane and others involved in splicing reactions. Such proteins have probably been secondarily recruited to mitochondria from genomes not necessarily related to that of the $\alpha$ proteobacterial ancestor.

The mitochondrial genome of the early diverging, freshwater protozoan Reclinomonas americana is more like that of a bacterium than any other mitochondrial genome sequenced so $\operatorname{far}^{38}$. This genome contains 67 protein-coding genes, most of which provide components of genetic processes and the bioenergetic system ${ }^{38}$. Several gene clusters in this mitochondrial genome are reminiscent of those in bacteria (Figs 5a, 6a). Most similarities represent retained, ancestral traits present in the common ancestor of bacteria and mitochondria. For example, the genes rplKAJL and $r p o B C$ are identically organized in $R$. prowazekii and the mitochondrial genome of Reclinomonas americana. Likewise, the genes encoding the S10, spc and the $\alpha$-ribosomal protein operons are organized similarly in the two genomes. The immediate proximity of these two clusters in the Reclinomonas americana mitochondrial DNA is reminiscent of the arrangement in free-living bacteria, whereas the physical separation of the two clusters in the $R$. prowazekii genome is atypical. A further rearrangement event is indicated by the fact that the rpsLrpsGfus cluster is located upstream of the rplKAJLrpoBC cluster in $R$. prowazekii, rather than downstream as it is in the Reclinomonas americana mtDNA. Phylogenetic reconstructions based on ribosomal proteins within each of these two clusters indicate that there is a close evolutionary relationship between $R$. prowazekii and mitochondria (Fig. 5b).

Mitochondria and $R$. prowazekii have a similar repertoire of proteins involved in ATP production and transport, including genes encoding components of the TCA cycle, the respiratorychain complexes, the ATP-synthase complexes and the ATP/ADP translocases. There are some similarities in the gene orders of some functional clusters (Fig. 6a). There are also some rearrangements of clusters that are specific to Rickettsia. One example is the inversion of segments corresponding to nuoJKLM and nuoGHI. Another is the scattered displacement of genes involved in the biogenesis of cytochrome $c$. Nevertheless, phylogenetic reconstructions based on components of the NADH dehydrogenase complexes indicate that there is a close evolutionary relationship between $R$. prowazekii and mitochondria (Fig. 6b).

We have identified as many as five genes coding for ATP/ADP transporters, all of which are expressed (R.M.P. et al., unpublished observations). The Rickettsia ATP/ADP translocases are monomers with 12 transmembrane regions each, whereas the mitochondrial translocates are dimers with six transmembrane regions per dimer. We found no relationship between the primary structures of the mitochondrial and Rickettsia ATP/ADP translocases, indicating that these transport systems may have originated independently.

The study of the $R$. prowazekii genome sequence supports the idea that aerobic respiration in eukaryotes originated from an ancestor of the Rickettsia, as indicated previously by phylogenetic reconstructions based on the rRNA gene sequences ${ }^{7,9}$. Phylogenetic analyses of the pet $B$ and $\operatorname{cox} A$ genes indicate that the respiration systems of Rickettsia and mitochondria diverged $\sim 1,500-2,000$ million years ago $^{10}$, shortly after the amount of oxygen in the atmosphere began 
to increase. The finding that the ATP/ADP translocases in $R$. prowazekii and mitochondria are of different evolutionary origin is problematic (R.M.P. et al., unpublished observations). Free-living bacteria do not seem to have homologues of ATP/ADP translocases, which are found only in organelles and in two obligate intracellular parasites, Rickettsia and Chlamydia. Thus it is not known whether the original endosymbiont was capable of efficient exchange of adenosine nucleotides with its host cell. More detailed comparative analysis of the genomes of $\alpha$-proteobacteria may refine our understanding of the origins of mitochondria.

\section{Methods}

Genome sequencing. We prepared genomic DNA from the Madrid E strain of $R$. prowazekii, which was originally isolated in Madrid from a patient who died in 1941 with epidemic typhus. We propagated $R$. prowazekii in the yolk sac of embryonated hen eggs and purified DNA according to standard procedures ${ }^{39}$. We sequenced the $R$. prowazekii genome by a whole-genome shotgun approach in combination with shotgun sequencing of a selected set of clones from a cosmid library (A.Z. et al., unpublished observations). Genomic and cosmid DNA was sheared by nebulization to an average size of $\sim 2 \mathrm{~kb}$. The random fragments were cloned into a modified M13 vector using the double adaptor method $^{40}$. We collected 19,078 sequence reads during the random sequencing phase using Applied Biosystems 377 DNA sequencers (Perkin-Elmer).

The sequences were assembled and the consensus sequence was edited using the STADEN program ${ }^{41}$. We verified the structure of the assembled sequence by end-sequencing of 3-kb-insert $\lambda$ Zap II clones ${ }^{36}, 10-\mathrm{kb} \lambda$ clones and $30-\mathrm{kb}$ cosmid clones. More than $97 \%$ of the genome was covered by clones from the three different libraries (A.Z. et al., unpublished observations). Gaps between contigs were closed by direct sequencing of clones from the three libraries or of polymerase chain reaction (PCR) products. The final four gaps were closed by direct sequencing of PCR products generated with the Long Range PCR system (Gene Amp). Regions of ambiguity were identified by visual inspection of the assembly and resequenced. The final assembly contains $\sim 20,000$ sequences. The genome sequence has eightfold coverage on average and no single region has less than twofold coverage. We estimate the overall error frequency to be $<1 \times 10^{-5}$.

Informatics. Sequence analysis and annotation was managed by CapDB (T.S.-P. et al., unpublished observations). We identified open reading frames of more than 50 codons as genes on the basis of their characteristic patterns in nucleotide-frequency statistics ${ }^{14}$ using BioWish ${ }^{42}$. The identified genes were analysed using the program BLASTX ${ }^{43}$ to search for sequence similarities in EMBL, TREMBL, SwissProt and in-house databases. We identified tRNA genes with the program tRNA scan-SE ${ }^{44}$. Remaining frameshifts were considered to be authentic and annotated as pseudogenes. Families of paralogues were constructed using BLAST to search for sequence similarities within the $R$. prowazekii genome. Multiple alignments and phylogenetic trees for genes with significant sequence similarities to genes in the public databases were constructed automatically using CLUSTAL-W ${ }^{45}$, Phylo_win ${ }^{46}$ and GRS $^{47}$. The final annotation was based on manual inspection of the phylogenetic placement of $R$. prowazekii in the resulting gene trees.

Received 21 July; accepted 24 September 1998.

1. Gross, L. How Charles Nicolle of the Pasteur Institute discovered that epidemic typhus is transmitted by lice: reminiscences from my years at the Pasteur Institute in Paris. Proc. Natl Acad. Sci. USA 93, 10539-10540 (1996).

2. Weisburg, W. G., Woese, C. R., Dobson, M. E. \& Weiss, E. A common origin of Rickettsiae and certain plant pathogens. Science 230, 556-558 (1985).

3. Woese, C. R. Bacterial evolution. Microbiol. Rev. 51, 221-227 (1987)

4. Weisburg, W. G. et al. Phylogenetic diversity of the rickettsias. J. Bacteriol. 171, 4202-4206 (1989).

5. Yang, D., Oyaizu, Y., Oyaizu, H., Olsen, G. J. \& Woese, C. R. Mitochondrial origins. Proc. Natl Acad. Sci. USA 82, 4443-4447 (1985).

6. Gray, M. W., Cedergren, R., Abel, Y. \& Sankoff, D. On the evolutionary origin of the plan mitochondrion and its genome. Proc. Natl. Acad. Sci. USA 86, 2267-2271 (1989),

7. Olsen, G. J., Woese, C. R. \& Overbeek, R. The winds of (evolutionary) change: breathing new life into microbiology. J. Bacteriol. 176, 1-6 (1994).

8. Viale, A. \& Arakaki, A. K. The chaperone connection to the origins of the eukaryotic organelles. FEBS Lett. 341, 146-151 (1994).
9. Gray, M. W. \& Spencer, D. F. in Evolution of Microbial Life (eds Roberts, D. M., Sharp, P. M., Alderson, G. \& Spencer, D. F.) 109-126 (Cambridge Univ. Press, Cambridge, 1996).

10. Sicheritz-Pontén, T., Kurland, C. G. \& Andersson, S. G. E. A phylogenetic analysis of the cytochrome $b$ and cytochrome $c$ oxidase I genes supports an origin of mitochondria from within the Rickettsiaceae. Biochim. Biophys. Acta 1365, 545-551 (1998).

11. Margulis, L. Origin of Eukaryotic Cells (Yale Univ. Press, New Haven, 1970).

12. Kurland, C.. G. Evolution of mitochondrial genomes and the genetic code. Bioessays 14, 709-714 (1992).

3. Andersson, S. G. E. \& Kurland, C. G. Reductive evolution of resident genomes. Trends Microbiol. 6, 263-268 (1998).

14. Andersson, S. G. E. \& Sharp, P. M. Codon usage and base composition in Rickettsia prowazekii. J. Mol. Evol. 42, 525-536 (1996).

15. Fleischmann, R. D. et al. Whole-genome random sequencing and assembly of Haemophilus influenzae Rd. Science 269, 496-511 (1995).

16. Fraser, C. M. et al. The Mycoplasma genitalium genome reveals a minimal gene complement. Science 270, 397-403 (1995).

17. Himmelreich, R. et al. Complete genome sequence analysis of the genome of the bacterium Mycoplasma pneumoniae. Nucleic Acids Res. 3, 109-136 (1996).

18. Fraser, C. M. et al. Genomic sequence of a Lyme disease spirochaete, Borrelia burgdorferi. Nature 390, 580-586 (1997).

19. Tomb, J.-F. et al. The complete genome sequence of the gastric pathogen Helicobacter pylori. Nature 388, 539-547 (1997).

20. Blattner, F. R. et al. The complete genome sequence of Escherichia coli K-12. Science 277, 1453-1462 (1997).

21. Deckert, G. The complete genome of the hyperthermophilic bacterium Aquifex aeolicus. Nature 392, 353-358 (1998).

22. Cole, S. T. et al. Deciphering the biology of Mycobacterium tuberculosis from the complete genome sequence. Nature 393, 537-544 (1998).

23. Fraser, C. M. et al. Complete genome sequence of Treponema pallidum, the syphilis spirochete. Science 281, 375-388 (1998).

24. Lobry, J. R. Asymmetric substitution patterns in the two DNA strands of bacteria. Mol. Biol. Evol. 13, 660-665 (1996).

25. Ebeling, S., Kündig, C. \& Hennecke, H. Discovery of a rhizobial RNA that is essential for symbiotic root nodule development. J. Bacteriol. 173, 6373-6382 (1991).

26. Koonin, E. V. \& Aravind, L. Genomics: re-evaluation of translation machinery evolution. Curr. Biol. 8, 266-269 (1998).

27. Curnow, A. W., Ibba, M. \& Soll, D. tRNA-dependent asparagine formation. Nature 382, 589-590 (1996).

28. Christie, P. J. Agrobacterium tumefaciens T-complex transport apparatus: a paradigm for a new family of multifunctional transporters in eubacteria. J. Bacteriol. 179, 3085-3094 (1997).

29. Melly, M. A., Duke, L. J., Liau, D.-F. \& Hash, J. H. Biological properties of the encapsulated Staphylococcus aureus. M. Infect. Immun. 10, 389-397 (1974).

30. Peterson, P. K., Wilkinson, B. J., Kim, Y., Schmeling, D. \& Quie, P. G. Influence of encapsulation on staphylococcal opsonization and phagocytosis by human polymorphonuclear leukocytes. Infect. Immun. 19, 943-949 (1978).

31. Lin, W. S., Cunnen, T. \& Lee, C. Y. Sequence analysis and molecular characterization of genes required for the biosynthesis of type I capsular polysaccharide in Staphylococcus aureus. J. Bacteriol. 176, 70057016 (1994).

32. Felsenstein, J. The evolutionary advantage of recombination. Genetics 78, 157-159 (1977).

33. Ohta, T. \& Kimura, M. On the constancy of the evolutionary rate of cistrons. J. Mol. Evol. 1, 18-25 (1971).

34. Ohta, T. Evolutionary rate of cistrons and DNA divergence. J. Mol. Evol. 1, 150-157 (1972).

35. Andersson, J. O. \& Andersson, S. G. E. Genomic rearrangements during evolution of the obligate intracellular parasite Rickettsia prowazekii as inferred from an analysis of 52015 bp nucleotide sequence. Microbiology 143, 2783-2795 (1997).

36. Andersson, S. G. E., Zomorodipour, A., Winkler, H. H. \& Kurland, C. G. Unusual organization of the rRNA genes in Rickettsia prowazekii. J. Bacteriol. 177, 4171-4175 (1995).

37. Andersson, S. G. E. \& Kurland, C. G. Genomic evolution drives the evolution of the translation system. Biochem. Cell Biol. 73, 775-787 (1995).

38. Lang, B. F. et al. An ancestral mitochondrial DNA resembling a eubacterial genome in miniature. Nature 387, 493-497 (1997).

39. Winkler, H. H. Rickettsia permeability: an ATP/ADP transport system. J. Biol. Chem. 251, 389-396 (1976).

40. Andersson, B. et al. A 'double adaptor' method for improved shotgun library construction. Anal. Biochem. 236, 107-113 (1996).

41. Staden, R. The Staden sequence analysis package. Mol. Biotech. 5, 233-241 (1996).

42. Sicheritz-Pontén, T. BioWish: a molecular biology command extension to Tcl/Tk. Comput. Appl. Biosci. 13, 621-622 (1997).

43. Altschul, S. F., Gish, W., Miller, W., Myers, E. W. \& Lipman, D. J. Basic local alignment search tool. J. Mol. Biol. 215, 403-410 (1990).

44. Low, T. M. \& Eddy, S. R. tRNAscan-SE: a program for improved detection of transfer RNA genes in genomic sequence. Nucleic Acids Res. 25, 955-964 (1997).

45. Thompson, J. D., Higgins, D. G. \& Gibson, T. J. CLUSTAL W: improving the sensitivity of progressive multiple alignment through sequence weighting, position-specific gap penalties and weight matrix choice. Nucleic Acids Res. 22, 4673-4680 (1994).

46. Galtier, N., Gouy, M. \& Gautier, C. SeaView and Phylo_win, two graphic tools for sequence alignment and molecular phylogeny. Comput. Appl. Biosci. 12, 543-548 (1996).

47. Sicheritz-Pontén, T. \& Andersson, S. G. E. GRS: a graphic tool for genome retrieval and segment analysis. Microb. Comp. Genomics 2, 123-139 (1997).

Acknowledgements. We thank C. Woese for discussions; M. Andersen for computer system support; and B. Andersson, K. Andersson, I. Tamas, B. Canbäck, A. Jamal, H. Amiri and S. Jossan for technical advice and assistance. This work was supported by the Swedish Foundation for Strategic Research, the Swedish Natural Sciences Research Council, the Knut and Alice Wallenberg Foundation and the European Commission.

Correspondence and requests for materials should be addressed to C.G.K. (e-mail: chuck@xray. bmc.uu.se). 


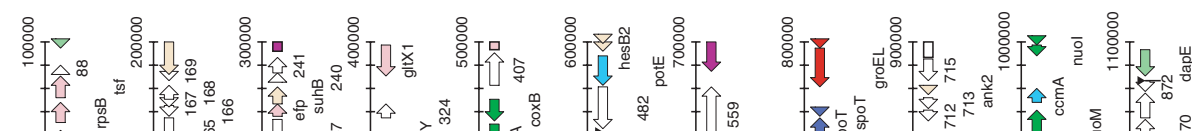

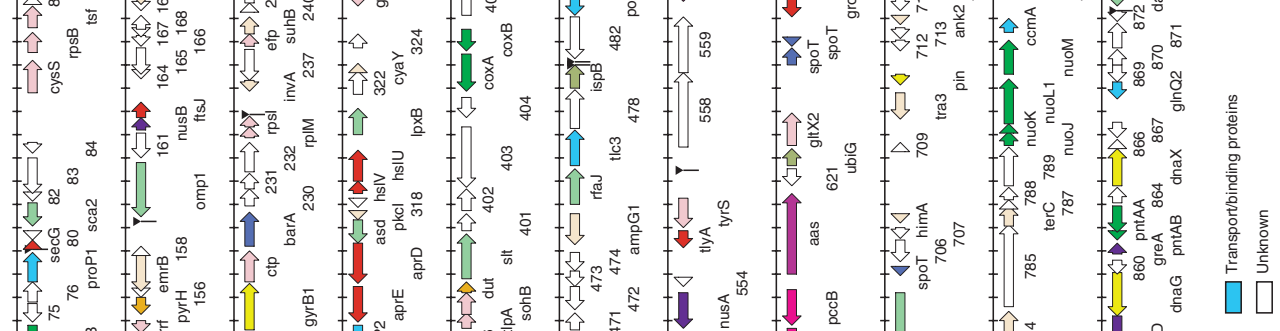

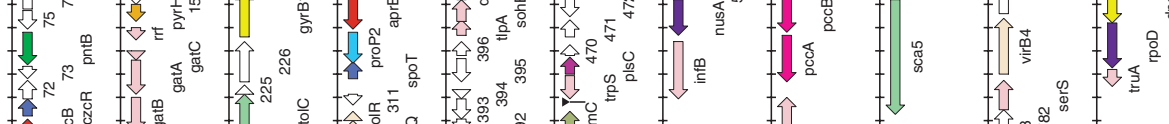

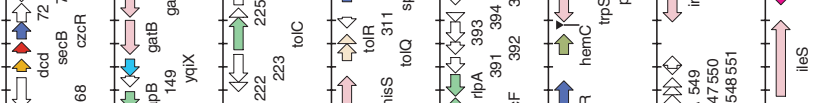

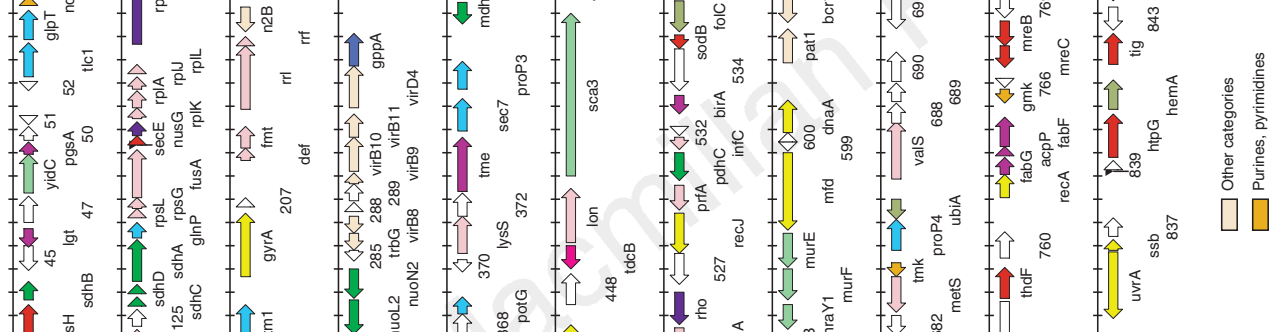

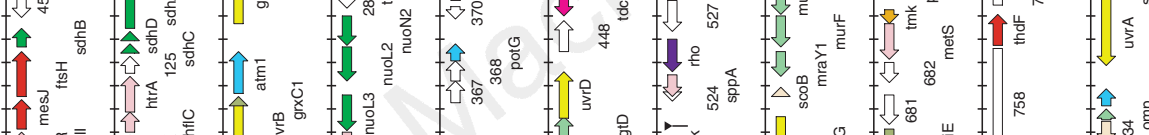

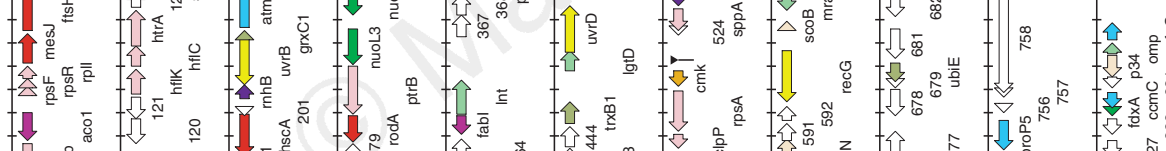

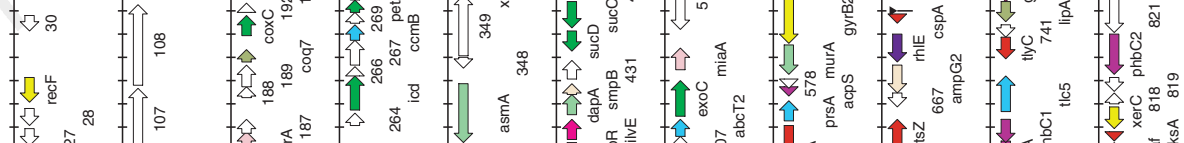

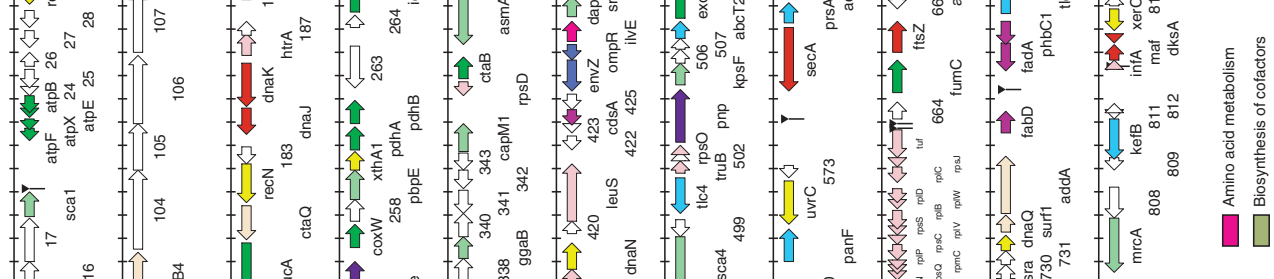

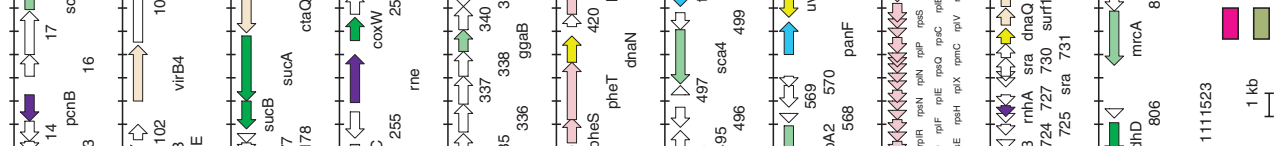

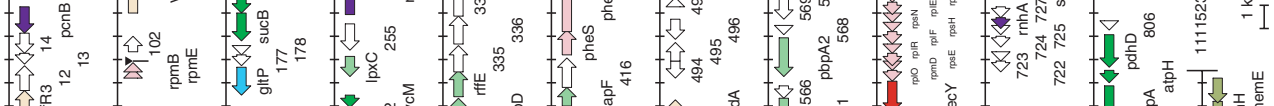

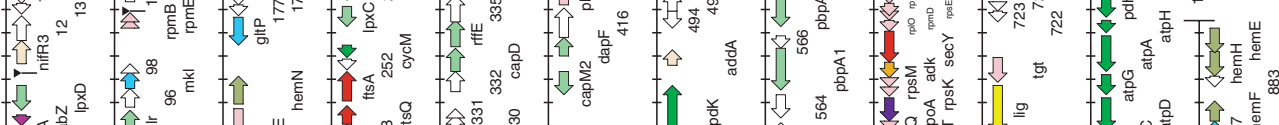

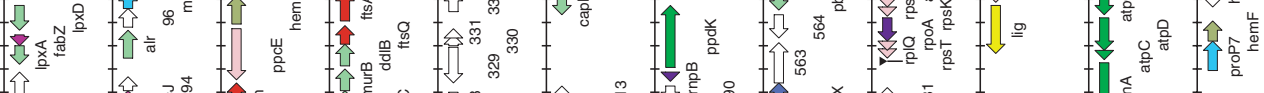

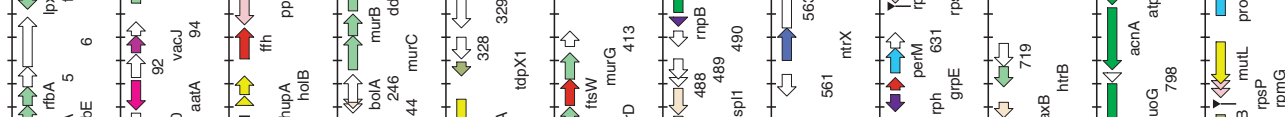

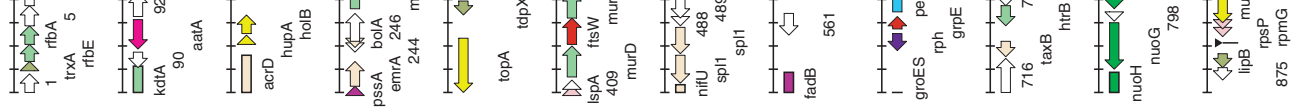


Table 1. Functional classification of Rickettsia prowazekii protein-coding genes. Gene numbers correspond to those in Fig. 2. Percentages represent per cent identitites.
AMINO ACID METABOLIS
AP743 glyA serine hydroxymethyl transferase (B-Mex, $60.9 \%$
RP428 ilvE branched-chain amino acid aminotransterase (B- Eco, $36.8 \%$ )
Amino acid degradation
RP091 aatA aspartate aminotransferase (B-Rme, 55.6\%)
RP618 pccA propionyl-CoA carboxylase $\alpha$ chain (E-Rno,

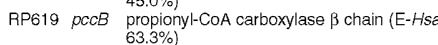
RP449 tdcB threonine dehydratase (E-Yeast, 35.3\%)
BIOSYNTHESIS OF COFACTORS...............25

Folic acid

RP536 folC folylpolyglutamate synthetase (B-Bsu, 34.5\%)
RP515 fold

RP383 folE GTP cyclohydrolase I (B-Syn, $48.1 \%$ )

Haem and porphyrins

RP841 hemA delta-aminolevulinate synthase (B-Bja, 49.1\%)

RP539 hemB delta-aminolevulinate dehydratase (B-Bja, 53.3\%)

RP885 hemE uroporhyrinogen decarboxylase (B-Aca, $426 \%$ )

RP885 hemE uroporhyrinogen decarboxylase (B-Rca, 42.6\%)
RP882 hemF coproporhyrinogen III oxidase (E-Gma, 43.0\%)

RP884 hemH ferrochelatase (B-Syn, 40.4\%)

RP847 hemK protoporphyrinogen oxidase (B-Eco, $44.3 \%$ )

RP175 hemN oxygen-independent coproporphyrinogen II (B-

Lipoate

$\begin{array}{ll}\text { RP742 lipA } & \text { lipoic acid synthetase (B-Hin, } 50.5 \% \\ \text { RP876 lipB } & \text { lipoic acid ligase (B-Mtu, 35.6\%) }\end{array}$

Menaquinone and ubiquinonens

RP190 coq7 ubiquinone biosynthesis prt Coq7 (E-Rno,

RP479ispB $\begin{array}{ll}36.9 \%) \\ \text { octaprenyl-diphosphate synthase (B-Eco, }\end{array}$

RP686 ubiA 4-hydroxybenzoate octaprenyltransferase (B-

RP541 ubiX $\quad \begin{aligned} & \text { Eco, 36.1\%) } \\ & \text { 3-octaprenyl-4-hydroxybenzoate carboxylase (B- }\end{aligned}$

RP680 ubiE $\begin{aligned} & E c 0,53.2 \%) \\ & \text { ubiquinone biosynthesis methyltransferase (B- }\end{aligned}$

RP622 ubiG $\begin{aligned} & E c 0,44.0 \%) \\ & 3 \text {-demethylubiquinone methyltransferase (B-Eco, }\end{aligned}$ $39.1 \%)$

Thio- and glutaredox

RP204 grxC1 glutaredoxin 3 (B-Eco, $50.0 \%$ )

RP745 grxC2 glutaredoxin 3 (B-Syn, 50.0\%)
RP327 tdpX1 thioredoxin-peroxidase (B-Hpy, 54.0\%)

RP445 $t x x B 1$ thioredoxin reductase (B-Hin, $52.0 \%$ )
RP514 $t x \times B 2$ thioredoxin reductase (B-CPa, 28.4\%)

CELL EnVELoPE $\ldots \ldots \ldots \ldots \ldots \ldots \ldots \ldots \ldots \ldots$

Diaminopimelate

$\mathrm{RP} 316$ asd aspartate-semialdehyde dehydrogenase (B- Vch

RP429 dapA dihydrodipicolinate synthase (A-Mja, 39.6\%)

RP148 dapB dihydrodipicolinate reductase (B-Hpy, $37.7 \%)$
RP194 dapD
tetrahydrodipicolinate N-succinyltransterase (B-

RP874 dapE $\begin{aligned} & \text { Eco, } 57.9 \% \text { ) } \\ & \text { succinyl-diaminopimelate desuccinylase (B-Hin, }\end{aligned}$

RP415 dapF diaminopimelate epimerase (B-Hin, $35.0 \%$ )

Membranes and lipoproteins
RP347 asmA outer membrane assembly protein (B-Eco,

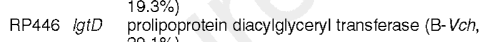

RP366 Int apolipoprotein N-acyltransferase (B-Hin, 29.1\%)

RP300 nlpD lipoprotein (B-Hin, 22.4\%)
RP390 ripA
rare lipoprotein A (B-Hin, 23.9\%)

RP224 tolC outer membrane protein (B-Eco, 22.9\%)

RP048 yidC inner membrane protein, $60 \mathrm{kDa}(\mathrm{B}-\mathrm{Hin}, 30.4 \%)$

Murein sacculus

RPO95 air alanine racemace (B-Hin, 29.5\%)

AaCF penicillin binding protein precursor (B-Bsu,

RP249 ddiB $\quad 33.8 \%$-alanine:D-alanine ligase (B-Hin, 32.8\%)

RP249 ddiB D-alanine:D-alanine ligase (B-Hin, $32.8 \%$ )
RP454 gimU UDP-N-acetylglucoseamine pyrophosphorylase

RP595 mraY1 phospho-N-acetylmuramoyl-pentapeptide-trans-

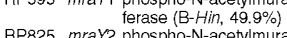

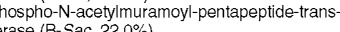

RP807 mrcA ferase (B-Sac, 22.0\%)

RP807 mrCA penicilin binding protein $1 \mathrm{~A}(\mathrm{~B}-\mathrm{E} C \mathrm{C}, 35.6 \%)$
RP579 murA UDP-N-acetylglucosamine 1 -carboxyvinyltrans-
ferase (B-Aca, 51.6\%)

RP248 murB UDP-N-acetylenolyruvoylglucosamine reductase

RP247 murC (B-Bsu, 35.7\%)

RP410 murD UDP-N-acetylmuramoylalanine-D-glutamate lig-

ase (B-Hin, 32.9\%)
RP597 murE UPD-MurNac-tripeptide synthetase (B-Bsu,

RP596 murF UDP-MurNac-pentapeptide synthetase (B-Eco,

RP412 murG UDP-MurNac-pentapeptide transterase (B-Bsu,

RP565 pbpA1 penicilin binding protein (B-Hin, $34.3 \%$ )
RP567 pbpA2 penicillin binding protein (B-Bsu, $30.7 \%$ )

RP567 pbpA2 penicillin binding protein (B-Bsu, 30.7\%)

RP259 pbpE penicillin binding protein (B-Bsu, 25.2\%)
RP400 sit lytic murein transglycosidase (B-Hin, 21.4\%) Surface polysaccharides, lipopolysaccharides and antigens
RP333 capD capsular polysaccharide biosynthesis protein CapD (B-Sau, 34.6\%)
RP344 capM1 capsular polysaccharide biosynthesis protein RP414 CapM (B-Sau, 24.9\%)

RP414 capM2 capsular polysaccharide biosynthesis protein

RP339 ggaB $\begin{aligned} & \text { CapM prt (B-Sau, 23.7\%) } \\ & \text { galactosamine-containing techoic acid biosyn- }\end{aligned}$

$\begin{array}{ll}\text { RP718 hitrB thesis (B-Bsu, 23.3\%) } & \text { lauroyl acetyltransterase (B-Hin, 21.5\%) }\end{array}$

RP007 IpXA UDP-N-acetylglucosamine acyltransterase (B- $\begin{array}{ll}\text { RP321 ipxB } & \text { Rri, 90.4\%) } \\ \text { lid A disaccharide synthetase (B-Hin, 27.3\%) }\end{array}$

RP254 ipxC UDP-3-O-acyl N-acetylglucosamine deacetylase RP009 ipXD UDP-3-O-(R-3-hydroxymyristoy)-glucosamine NRP062 kdsA $\begin{aligned} & \text { acetyltransferase (B-Rri, 92.4\%) } \\ & \text { 3-deoxy-d-manno-octulosonic acid 8-phosphate }\end{aligned}$ RP379 $k d s B$ STP:CMP-3-deoxy-manno-octulosonate-cytidylyl RP089 kdtA transferase (B-Hin, 34.8\%) (B-ECO, 28.9\%)

RP505 kps F polysiliac acid capsule expression protein (B-

RP833 omp cell surface antigen, $17 \mathrm{kD}$ (B-Rty, 46.9\%)

RP771 pal peptidoglycan-associated lipoprotein (B-Eco,

RP476 rfaJ lipopolysaccharide 1,2-glucosyltransterase (B-

RP004 rfbA Sty $\quad$-antigen export system permease (B-Kpn, $22.3 \%$ )

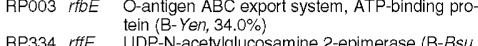

$\begin{array}{ll}\text { UDP-N-acetylglucosamine 2-epimerase } & 26.8 \%) \\ \text { RP018 sca1 } & \text { cell surface antigen (B- } R r i, 24.9 \% \text { ) }\end{array}$

RP081 sca2 cell surface antigen (B-Rri, 27.4\%

$\begin{array}{lll}\text { RP451 } & \text { scas } & \text { cell surface antigen (B-Rri, } 27.6 \% \\ \text { RP498 sca4 } & \text { cell surface antigen (B-Pja, } 67.4 \%\end{array}$

RP779 udg UDP-glucose 6-dehydrogenase (B-Pae, 31.8\%)

CELLULAR PROCESSES $\ldots \ldots \ldots \ldots \ldots \ldots \ldots \ldots, 44$

Cell division
RP251 fisA cell division protein FtsA (B-Hin, 29.5\%)

RPO43 ftsH cell division protein FtsH (B-Eco, 54.0\%)

RP823 ftsK cell divion protin FtSK (B-Cb, 41.5\%)

RP250 fisQ cell division protein FtsQ (B-Hin 17.9\%)

RP411 ftsW cell division protein FtsW (B-Eco, 33.2\%

$\begin{array}{ll}\text { RP775 fts Y } & \text { cell division protein FtsY (B-Hin, } 43.2 \% \text { ) } \\ \text { RP666 fisZ cell division protein FtsZ (B-Wsp. } 65.3 \%\end{array}$

RP056 gidA glucose inhibited division protein A (B-Eco, 48.8\%)

RP057 gidB glucose inhibited division protein (B-Ppu, 26.8\%)

RP042 mesJ cell cycle protein MesJ (B-ECO, 22.1\%)

RP768 mreB rod shape-determining protein (B-Eco, $60.5 \%$ )

RP767 mreC rod shape-determining protein (B-Eco, $23.1 \%$ )
RP280 rodA rod shape-determining protein (B-Eco, 38.1\%)

Cell killing

RP555 thyA hemolysin (B-Thy, 34.3\%)
RP740 thyC hemolysin (B-Thy, 28.8\%)

RP670 CSPA cold shock protein (B-Scl, $57.6 \%$ )

RP816 dksA DnaK suppressor protein (B-Hin, 38.8\%)

RP184 dnas heat shock protein (B-Bov, 49.7\%)

RP185 dhax heal shock protein 70 (B-Rme, 72.7\%)

RP627 groEL heal shock protein GroEL (B-Ame, 69.4\%)

RP629 groE heat shock protein GrpE (B-CCr. $39.5 \%)$

RP200 hscA heat shock protein A (B-Hin, 39.6\%)

RP319 hslV heat shock prt HsIV (B-Hin, 54.1\%)

RP273 hsp22 heat shock protein (E-Phy cP, 29.0\%)
RP840 $h t p G$ heat shock protein C62.5 (B-ECO, 43.1\%)

Detoxification

RP535 sodB superoxide dismutase (B-Lpn, $53.4 \%$ )
RP759 thdF thiophene and furan oxidizer (B-Hin, 34.7\%)

Protein and peptide secretion

RP315 aprD protease secretion ATP-binding protein (B-Pae

RP314 aprE $\begin{aligned} & 40.0 \% \text { ) } \\ & \text { protease secretion ATP-binding protein (B-Pae, }\end{aligned}$

$\begin{array}{lll}\text { RP173 ffh } & \begin{array}{l}32.4 \% \text { signal recognition particle receptor protein (B-ECo, } \\ 49.6 \% \text { ) }\end{array}\end{array}$

RP275 lepA $\quad \begin{aligned} & 49.6 \% \\ & \text { GTP-binding membrane protein (B-Bsu, } 57.0 \% \text { ) }\end{aligned}$

RP116 lepB $\quad$ signal peptidase (B-Sty, $37.3 \%)$
RP575 SecA preprotein translocase SecA subunit (B-Rca, $51.8 \%)$

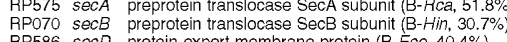

RP134 secE preprotein translocase SecE subunit (B-Bsu, 37.3\%)

RP114 secF protein-export membrane protein (B-Hin, 37.7\%)

RP079 secG protein-export membrane protein (B-Hpy, 32-0\%)

$\begin{array}{lll}\text { RP639 secY } & \begin{array}{l}\text { preprotein translocase SecY subu } \\ \text { trigger factor (B-Eco, 32.0\%) }\end{array}\end{array}$

ENERGY METABOLISM

...67

ATP-proton motive force interconversion

RP803 atpA ATP synthase F1 alpha subunit (B-Rru, 66.2\%)

RP023 atpB ATP synthase Fo subunit a (B-Rru, $51.5 \%$ )
RP800 atpC ATP synthase F1 epsilon subunit (B-Aru, 24.5\%)

RPso alpC ATP synthase F1 epsilon subunit (B-Aru, 24.5\%)

RP801 atpD ATP synthase F1 beta subunit (B-Aca, 77.0\%)

RPO20 atpF ATP synthase FO subunit b (B-Rru, 21.1\%)

RP802 atpG ATP synthase F1 gamma subunit (B-Rbi, $38.0 \%$ )

RP804 atpH ATP synthase F1 delta chain (E-Osicp 26.4\%)
RP021 atpX ATP synthase FO subunit b' (E-Ram mt, 28.6\%)

Electron transport

RP588 ccmE cytochrome $c$ biogenesis protein (B-Hin, $33.2 \%$ )

RP703 ccmF cytochrome c biogenesis protein (E-Ram mt, 33.6\%)

RP406 coxB cytochrome coxidase subunit II (E-Mpo mt, $48.0 \%$ )

RP191 coxC cytochrome $c$ oxidase subunit III (E-Pwi mt, $48.9 \%$ )

RP257 coxW cytochrome coxidase assemly ( $\mathrm{E}-\mathrm{Sce}, 35.2 \%$ )

RP304 cox11 cytochrome coxidase assembly (E-Ram mt, 42.9\%)
RP346 ctaB cytochrome coxidase assembly factor (B-Pde,

cytochrome coxidase asse

RP216 cydA cytochrome oxidase $d$ subunit I (B-Avi, $34.0 \%$ )

RP217 cydB cytochrome oxidase $d$ subunit II (B- $E C O, 30.0 \%$ )

RP272 fbch ubiquinol cytochrome $c$ oxidoreductase,

RP829 foxA ferredoxin (Rca, 57.5\%)

RP357 nUOA NADH dehydrogenase I chain A (E-Pwi mt, $64.6 \%$ )

AADH dehydrogenase I chain B (E-Ram $\mathrm{mt}, 73.2 \%$ )

RP355 nuoC NADH dehydrogenase I chain C (B-Pde, 42.1\%)

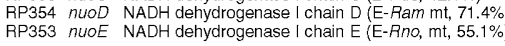

RP115 nuoF NADH dehydrogenase I chain $F$ (B-Pde, 69.1\%) RP796 nuot NADH dehydrogenase I chain H (E-Ram mt, $63.5 \%$ RP795 nuol NADH dehydrogenase I chain I (E-Ram $\mathrm{mt}, 71.4 \%$ ) RP790 nuoJ NADH dehydrogenase I chain J (E-Ram mt 42.3\%) RP791 nuoK NADH dehydrogenase I chain K (B-Pde, 61.4\%)
RP792 nuoL1 NADH dehydrogenase I chain L (E-Ram mt, 45.5\%) RP283 nuoL2 NADH dehydrogenase I chain $\mathrm{L}$ (E-Aja cp, 27.0\%) RP282 nuoL3 NADH dehydrogenase I chain $L$ ( $(E-A m e m t, 17.0 \%$ )

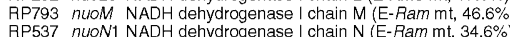
RP537 nuoN1 NADH dehydrogenase I chain N (E-Ram mt, 34.6\%)
RP284 nuoN2 NADH dehydrogenase I chain N (E-Ram mt, $23.2 \%$ ) RP284 nuoN2 NADH dehydrogenase I chain N (E-Pam mt,
RP270 petA Rieske-l iron sulphur protein (B-Bja, $58.3 \%$ ) RP270 petA Rieske-l iron sulphur protein
RP271 petB cytochrome $b$ (B-Aru, $65.4 \%)$

RP271 petB cytochrome b (B-Rru, 65.4\%)
RP863 pntAA NAD(P) transhydrogenase $\alpha$ subunit (B-Eco, $37.7 \%)$ RP862 pntAB NAD(P) transhydrogenase a subunit (B-Hin, 44.7\%)
RP074 pntB NAD(P) transhydrogenase $\beta$ subunit (B-Hin, $51.5 \%$ ) Fermentation

RP110 ackA acetate kinase (B-Cts, 38.4\%)

Giycolysis
RP492 ppdK pyruvate, orthophosphate dikinase (E-Ftr, 48.8\%)

Phosphate
AP589 ppa inorganic pyrophosphatase (B-Eco, $59.3 \%$ )

Pyruvate dehydrogenase
RP261 pdhA pyruvate

RP261 pdhA pyruvate dehydrogenase E1 component, $\alpha$ sub-

RP262 pdhB pyruvate dehydrogenase $\mathrm{E} 1$ component, $\beta$ sub-

RP530 pdhC dihydrolipoamide acetyltransferase E2 compo-

RP460 pdho nent (E-Ano, 45.1\%)

RP460 pdhD dihydrolipoamide dehydrogenase E3 component

RP805 pdhD dihydrolipoamide dehydrogenase E3 component

RP799 acnA aconitate hydratase (B-Lpn, 59.1\%)

RP665 fumC fumarate hydratase (B-Ror, 63.5\%

$\begin{array}{lll}\text { RP844 } & \text { gltA } & \text { citrate synthase (B-Rty, } 97.8 \%) \\ \text { RP265 } & \text { icd } & \text { isocitrate dehydrogenase (B-Tth, 38.6\%) }\end{array}$

RP376 mdh malate dehydrogenase (B-Can, $51.5 \%$ )
RP128 sdhA succinate dehydrogenase, flavoprotein subunit

$\begin{aligned} \text { RP044 } \operatorname{sdhB} B & \begin{array}{l}\text { (B-Bja, } 70.0 \% \text { ) } \\ \text { succinate dehydrogenase, iron-sulphur protein (E- } \\ \text { Ram } \mathrm{mt}, 69.0 \% \text { ) }\end{array}\end{aligned}$

RP126 solhC $\begin{gathered}\text { succinate dehydrogenase, cytochrome } b_{556} \\ \text { subunit (E-Ram mt, } 39.5 \%)\end{gathered}$

RP127 soho $\begin{aligned} & \text { subunit (E-Ram } \mathrm{mt}, 39.5 \% \text { ) } \\ & \text { succinate dehydrogenase, subunit IV (E-Ram }\end{aligned}$

$\begin{array}{ll}\text { RP180 sucA } & \mathrm{mt}, 25.6 \% \text { ) } \\ 2 \text {-oxoglutarate dehydrogenase (B-Hin, 44.3\%) }\end{array}$ RP179 SUCB dihydrolipoamide succinyltransferase (B-ECO, 48.7\%)

RP433 sucC succinyl-CoA synthetase, $\beta$ subunit (B-Eco, $52.1 \%$ )
RP432 sucD succinyl-CoA synthetase, $\alpha$ subunit (E-Ddi, 70.7\%)

Sugars

RP509 exoC phosphomannomutase (B-Abr, 42.7\%)

FATTY ACID AND PHOSPHOLIPID METABOLISM. . . . 25

RP620 aas 2-acyl-glycerol-phosphate-ethanolamine (B-Eco,

RP038 aco1 acyl-CoA desaturase (E-Yeast, 27.6\%)

RP763 acpP acyl carrier protein (B-Lmu, 52.6\%)

RP577 acpS holo-[acyl carrier protein] synthase (B-Eco, 38.5\%)

RP533 birA biotin Ac-CoA carboxylase synthase (B-Pde, $33.6 \%$ )

RP735 fabD malonyl-COA:Acyl carrier protein transacylase

RP764 fabF $\begin{aligned} & \text { (B-Bsu, } 40.3 \% \text { ) } \\ & 3 \text {-oxoacyl-[acyl-carrier-protein] synthase II(B- }\end{aligned}$

RP762 fabG $\begin{array}{ll}\text { Eco, } 53.5 \% \text { ) } & \text { 3-oxoacyl-[acyl-carrier-protein] reductase (B- }\end{array}$

RP772 fabH 3 -oxoacyl-[acyl-carrier-protein] synthase (B-

RP365 fabl enoyl-[acyl-carrier-protein] reductase (B-Asp, 49.0\%)

RP008 fabZ 3R-hydroxymyristoyl acyl carrier protein dehy

RP737 fadA dratase (B-Ari, 91.7\%)

RP560 $\mathrm{fadB}$ fatty oxidation complex a subunit (E-Cel, $30.6 \%)$

RP442 gpsA glycerol-3-phosphate dehydrogenase (B-ECO,

RP046 lgt prolipoprotein diacylglycerol (B-Eco, 39.1\%)

RP035 phbB acetoacetyl-CoA reductase (B-Zra, $52.9 \%$ )
RP738 phbC1 poly-beta-hydroxybutyrate polymerase (B-CVi,

$22.7 \%$ )
RP820 phbC2 poly-beta-hydroxybutyrate polymerase (B-Mex,

RP750 pgpA phosphatidylglycerophosphatase A (B-Hin, 31.9\%)

RP049 pgsA phospatidylglycerophosphate synthase (B-Bsu,

RP469 plsC 1-acyl-glycerol-3-phosphate acyltransferase (E-

RP242 pSsA phosphatidylserine synthase (B-Hpy, 28.8\%)

RP373 tme malic enzyme (B-Hin, $45.5 \%$ )
RP093 vacJ VacJ lipoprotein precursor (B-Sf, 33.8\%)

PURINES, PYRIMIDINES.

14

Deoxyribonucleotide metabolism

RP069 dcd deoxycytidine triphosphate deaminase (B-Hin,

RP064 dgtP dexyguanosine triphosphate triphosphohydro-

RP399 dut deoxyuridine 5 "triphosphate nucleotidohydro-

RP055 ndk lase (B-Hin, 43.1\%)

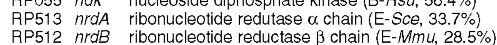

RP301 thy A thymidylate synthase (E-Ddi $47.9 \%$ )

RP684 tmk thymidylate kinase (B-Bsu, $38.7 \%$ )

Purine ribonucleotide metabolism

RP638 adk adenylate kinase (B-Sco, $33.8 \%$ )

$\begin{array}{lll}\text { RP765 gmk } & \text { guanylate kinase (B-Hin, } 49.0 \%) \\ \text { RP220 } & \text { purC } & \text { phosphoribosylaminoimidazole-succinocarbox- }\end{array}$ amide synthase (B-Spn, 34.0\%)

Pyrimidine ribonucleotide biosynthesis

RP522
cmk cytidylate kinase (B-Eco, 34.9\%)
RP378 pyrG CTP synthase (B-Abr. $54.7 \%)$ 
RP155 pyrH uridylate kinase (B-Syn, 53.3\%)

REGULATORY FUNCTIONS $\ldots \ldots \ldots \ldots \ldots \ldots \ldots \ldots 14$
RP229 barA histidine kinase sensor protein $(B-E C 0,23.2 \%)$ $\begin{array}{lll}\text { RP229 } & \text { barA } & \text { histidine kinase sensor } \\ \text { RP07 } & \text { transcriptional activator protein (B-Aeu, 35.1\%) }\end{array}$ RP426 envZ histidine $k$

RP294 gppA Sti, 23.65)

RP011 nifR3 transcriptional activator nitrogen assimilation

RP614 ntrY $\begin{aligned} & \text { protein (B-Abr, 59.0\%) } \\ & \text { histidine kinase nitrogen sensor protein (B-Aca, }\end{aligned}$

$30.6 \%)$
0

$X$ transcriptional activator nitrogen assimilation

RP427 OMpR transcriptional activator protein OmpR (B-Aca,

RP465 phoR histidine kinase phosphatase synthesis sensor

protein (B-Bsu, 24.4\%)
RP312 Spo
(p)ppGpp 3 - - pyrophosphohydrolase (B-Eco, 29.9\%)

RP624 SpoT* (p)ppGpp 3'-pyrophosphohydrolase (B-Mpn, 27.8\%)

RP705
spoT* (p)ppGpp 3'--pyrophosphohydrolase (B-ECo, $31.7 \%$ )
RP517 yhbH sigma 54 modulation protein (B-Bja, 26.2\%)

REPLICATION $\ldots \ldots \ldots \ldots \ldots \ldots \ldots \ldots \ldots \ldots \ldots, 46$

Degradation of DNA

RP734 addA ATP-dependent nuclease (B-Bsu, 23.7\%)

RP676 xthA2 exodeoxyribonuclease III (B-Eco, 30.1\%)

RP676 XthA2 exodeoxyribonuclease III (B-Eco, $33.2 \%)$
RP675 XseA
exodeoxyribonuclease large subunit (B- $E c 0,31.7 \%)$

RP350 XseB exodeoxyribonuclease small subunit (B-Eco, $32.5 \%$ )

DNA replication, restriction, modification, recombination and repair
RP601 dnaA chromosomal replication initiation protein DnaA (B-

RP601 dnaA chromosomal replication initiation pr
Eco, $44.1 \%$ )
RP542 dnaB

RP542 dnaB DNA helicase (E-Osi cp, 40.9\%)
RP778 dnaE DNA polymerase III alpha subunit (B-Sty, 37.2\%)

RP859 dnaG DNA primase (B-Sty, 29.0\%)

RP419 dnaN DNA polymerase III beta subunit (B-Ppu, 29.9\%)

RP732 dnaQ DNA polymerase III epsilon subunit (B-Sty, 46.7\%)

RP206 gyra DNA gyrase A subunit (B-Rsp, 49.4\%)

RP206 gyrA DNA gyrase A subunit (B-Asp, 49.4\%)
RP227 gyrB1 DNA gyrase B subunit (B-Sci, 42.0\%)

RP580 gyrB2 DNA gyrase B subunit (B-Ppu, 51.5\%)
RP172 holB DNA polymerase III, delta prime subunit (B-Pae,

$22.3 \%)$
RP171 hupA DNA binding protein $\mathrm{HU}(\mathrm{B}-\mathrm{V}$ pr, 47.8\%)

$\begin{array}{llll}\text { RP720 } & \mathrm{lig} & \text { DNA ligase (B-Zmo, 45.7\%) } \\ \text { RP777 } & \text { metK* } & \text { S-adenosylmethionine synthetase (B-Eco, 66.3\%) } \\ \text { RP598 } & \text { mfd transcription-repair coupling factor (B-Hin, 33.9\%) }\end{array}$

RP351 mpg DNA-3-methyladenine glycosidase (E-Hsa, 29.7\%)
RP880 mutl DNA mismatch repair protein MutL (B-Spn, 35.4\%)

RP298 mutS DNA mismatch repair protein MutS (B-Bsu, 39.0\%)

RP746 nth endonuclease III (B-ECO, $50.7 \%$ )
RP067 parC DNA topoisomerase IV subunit A (B-Hin, 39.0\%)

$\begin{array}{ll}\text { RP067 parC } & \text { DNA topoisomerase IV subunit A (B-Hin, } \\ \text { RP711 } & \text { pin } \\ \text { invertaselrecombinase (B-Eco, } 38.0 \% \text { ) }\end{array}$

RP540 priA primosomal protein replication factor (B-Aru $39.7 \%$ )

RP546 radA DNA repair (B-Bsu, 46.5\%)

RP761 recA recombination protein RecA (B- Pde, 71.2\%)

RPO29 recF DNA repair protein, ATP binding protein (B- $\mathrm{Cc}$,

$\begin{array}{ll} & 30.4 \% \text { ) } \\ \text { RP593 recG ATP-dependent DNA helicase (B-Eco, 34.1\%) }\end{array}$

RP528 reas single-stranded DNA-specific exonuclease (B-Eco,

RP182 recN recombination protein RecN (B-Hin, $31.6 \%$ )

RP438 rech recombination protein RecR (B-Bsu, 36.9\%)

RP385 ruvA Holliday junction DNA helicase (B-Pae, $35.0 \%$ )

RP386
ruVB Holliday junction DNA helicase (Pae, $51.5 \%$ )

RP119 rUVC Holliday junction endodeoxyribonuclease (B- $E C O$,

RP836 ssb single-stranded binding protein (B-Bab, 52.6\%)

$\begin{array}{lll}\text { RP835 } & \text { Uvr A } & \text { repair excision nuclease subunit A (B-Eco, } 57.7 \%) \\ \text { RP203 uvrB } & \text { repair excision nuclease subunit B (B-Hin, 56.0\%) }\end{array}$

RP572 UVrC repair excision nuclease subunit C (B-Pfl, $36.9 \%)$

RP447 uvrD DNA helicase (B-Sau, 43.5\%)

$\begin{array}{lll}\text { RP817 } & \text { xerC } & \text { integrase/recombinase (B-Bsu, } 32.2 \% \\ \text { RP361 } & \text { xerD } & \text { integrase/recombinase (B-Eco, } 37.6 \%\end{array}$

TRANSCRIPTION

Degradation of ANA

RP504 pnp polyribonucleotide nucleotidyltransferase (B-ECo,

RP117 rnc $\quad \begin{aligned} & 48.9 \%) \\ & \text { ribonuclease III (B-Hpy, 40.2\%) }\end{aligned}$

RP462 rnd ribonuclease D (B-Eco, 28.5\%)

$\begin{array}{lll}\text { RP256 } & \text { rne } & \text { ribonuclease E (B-Eco, 35.9\%) } \\ \text { RP726 } & \text { rnhA } & \text { ribonuclease HI (B-Msm, 43.4\%) }\end{array}$

RP202 $r$ hhB ribonuclease HII (B-Eco, 44.7\%)

$\begin{array}{lll}\text { RP628 } & \text { rph } & \text { ribonuclease PH (B-Hin, 55.05\%) }\end{array}$

RP861 greA transcription elongation factor GreA (B-Hin, 61.4\%) RP553 nusA transcription termination factor NusA (B-ECO,

RP162 nuSB transcription termination factor NusB (B-Eco,

RP135 nusG transcription antitermination protein NusG (B-Eco,

$\begin{array}{ll}\text { RP015 pcnB } & 42.2 \%) \\ \text { poly (A) polymerase I (B-Bsu, 26.3\%) }\end{array}$

RP669 thiE ATP-dependent RNA helicase (B-Eco, 38.3\%)
RP526 tho transcription termination factor Rho (B-Rsp, $72.0 \%$ )

RP635 rPOA RNA polymerase alpha subunit (B-Bpe, 47.2\%)

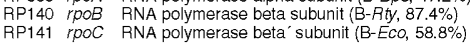

$\begin{array}{lll}\text { RP141 } & r \text { POC } & \text { RNA polymerase beta' subunit (B-ECO, } 58.8 \% \text { ) } \\ \text { RP303 } & r p 0 H & \text { RNA polymerase sigma-32 tactor (B-Atu, 52.0\%) }\end{array}$

TRANSLATION ........................118

Aminoacyl-tRNA synthetases

RP856 alaS alanyl-tRNA synthetase (B-Bba, 52.7\%)

RP065 argS arginyl-tRNA synthetase (B-Hpy, 33.0\%)

RP145 aspS aspartyl-tRNA synthetase (B-Syn, 43.3\%

RP325 gltX1 glutamyl-tRNA synthetase (B-Abr, 45.6\%)

RP623 gltX2 glutamyl-tRNA synthetase (B-Hoy, 40.3\%)

RP850 glyQ glycyl-tRNA synthetase (B-Mca, 60.6\%)

RP849 glys glycyl-tRNA synthetase (B-Bsu, 32.9\%)

RP620 hiles isoleucyl-tRAA synthetase (B-Mtu, 48.6\%)

RP371 lys S lysyl-tRNA synthetase (B-Bbu, 26.3\%)

lysyl-tANA synthetase (B-Bbu, 26.3\%)

RP683 metS methionyl-tRNA synthetase (B-Bsu, $46.9 \%)$
RP417 phes phenylalanyl-tRNA synthetase alpha sub (B-Hin,

RP418 pheT phenylalanyl-tRNA synthetase beta sub (B-Hin,
$33.9 \%)$

AP384 proS proline-tRNA synthetase (B-Zmo, $51.8 \%$ )

RP783 sers seryl-tRNA synthetase (B-Cbu, 47.2\%)

RP221 thrS
RP468 threonyl-tRNA synthetase (B-Hin, $50.6 \%$ )
tryptophanyl-tRNA synthetase (B-Syn, 48.5\%)

RP556 tyrs tyrosyl-tRNA synthetase (B-Bca, 38.7\%)

TRNA and amino acyl-tRNA modification

AP208 def methionyl-tRNA deformylase (B-Eco, 49.4\%)

RP209 $t m t$ methinoyl-tRNA formyltransterase (B-Hin, 41.9\%)

$\begin{array}{ll} & \text { glutamyl-tRNA (GIn) amidotransferase subunit A (B- } \\ \text { RP151 gatB } & \text { glutamyl-thNA (GIn) amidotransferase subunit B (B- }\end{array}$

RP153 gatC $\begin{aligned} & \text { Mca, 46.9\%) } \\ & \text { glutamyl-thNA (GIn) amidotransferase subunit C (B- } \\ & \text { Bsu, 24.7\%) }\end{aligned}$

RP672 $\mathrm{ksgA}$ dimethyladenosine transferase (B-Bsu, $35.7 \%$ )
RP510 miaA tRNA delta-2-isopentenylpyrophosphate (IPP) tran

$\begin{array}{ll}\text { RP605 pth } & \text { terase (B-Atu, 30.7\%) } \\ \text { peptidyl-thNA hydrolase (B-Hin, } 40.5 \% \text { ) }\end{array}$

RP213 queA S-adenosylmethionine:tRNA ribosyltransferase-iso

merase (B-Hin, $43.3 \%$ )
RP721 tgt tRNA-guanine transglycosylase (B-Zmo, 61.2\%)

$\begin{array}{ll}44.7 \%) & \text { ) } \\ & \end{array}$

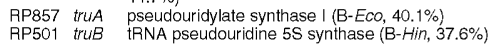

Degradation of proteins, peptides and glycopeptides

RP036 CIPB ATP-dependent protease, ATP binding subunit (B-

$\begin{array}{ll}\text { HP520 cipP AT, } 54.3 \% \text { ) } & \text { ATP-dependent Clp protease (B-Yen, } 67 \% \text { ) }\end{array}$

RP692 clpX ATP-dependent protease, ATPase subunit (B-Eco,

$\begin{array}{lll}\text { RP228 ctp } & 62.8 \% \text { ) } \\ \text { tail-specific protease precursor (B-Bba, } 42.6 \%)\end{array}$

$\begin{array}{lll}\text { RP037 gcp } & \text { sialoglycoprotein endopeptidase (B-Hin, 42.2\%) } \\ \text { RP123 hfiC } & \text { lambda cll stability-governing protein (B-Eco, 33.9\%) }\end{array}$

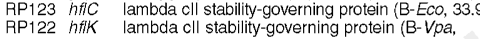

RP124 htra $\begin{array}{ll}30.3 \%) \\ \text { serine protease (B-Bhe, 37.7\%) }\end{array}$

$\begin{array}{ll}\text { RP186 htrA } & \text { protease DO (E-Sce, 26.7\%) } \\ \text { AP450 ion } & \text { ATP-dependent protease LA (B- } C c r, 53.1 \%)\end{array}$

$\begin{array}{ll}\text { RP450 ion } & \text { ATP-dependent protease LA (B-Ccr, } 53.1 \% \\ \text { RP408 ispa } & \text { lipoprotein signal peptidase (B-Bsu, 27.9\%) }\end{array}$

RP824 map methionyl aminopeptidase (B-Sty, 55.3\%)

RP142 pepA aminopeptidase A (B-Pae, 36.6\%)

AP174 ppcE peptidase II (B-Asn, 32.5\%)

RP281 ptrB protease II (B-ACC, 34.2\%)

RP398 sohB protease IV (B-Mja, 23.9\%)
RP525 sppA protease IV (B-Hin, 27.6\%)

Protein modification and translation factors

RP238 efp elongation factor $P$ (B-Bsu, $39.5 \%$

RP814 infA initiation factor IF-1 (B-Hin, 67.1\%

RP552 infB initiation factor IF-2 (B-Hin, 42.6\%

RP531 infC
initiation factor IF-3 (B-Pvil, $47.7 \%$ )
RP529 prfA peptide chain release factor RF-1 (B-Bsu, 50.1\%)

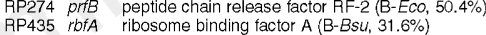

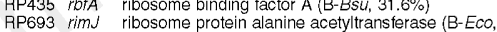

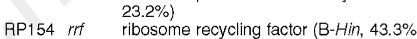

RP397 ttpA thiol:disulphide interchange protein (B-Bja, 27.4\%)

$\begin{array}{lll}\text { RP661 tuf } & \text { elongation factor Tu (B-TCu, 81.5\%) } \\ \text { RP087 tsf } & \text { elongation factor Ts (B-Sci, 40.7\%) }\end{array}$

Ribosomal proteins; synthesis and modification

RP137 rolA ribosomal protein L1 (B-Cgr, 50.2\%)

RP659 rpic ribosomal protein L3 (E-Sce, 44.1\%)

RP658 rplD ribosomal protein L4 (B-Bst, $39.3 \%)$

RP647 rple ribosomal protein L5 (B-Aco, 53.6\%)

$\begin{array}{ll}\text { RP644 } & \text { rplf r ribosomal protein L6 (B-BSt, 45.4\%) } \\ \text { RP041 rpll ribosomal protein L9 (E-Ppu cp, 33.6\%) }\end{array}$

AP136 rp/K ribosomal protein L11 (E-Ram mt, 45.5\%)

RP139 rpll ribosomal protein L7/L12 (B-Bab, 66.9\%)

AP233 roiM ribosomal protein L13 (B-Sca, 52.8\%)

RP649 rpiN ribosomal protein L14 (B-Eco, 69.6\%)

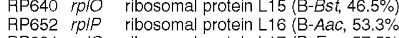

$\mathrm{AP652}$
$\mathrm{RP} 634$
$\mathrm{rpplQ}$

RP643 rplR ribosomal protein L18 (B-Bst, 58.6\%)

RP112 rplS ribosomal protein L19 (B-ECO, 58.8\%)

RP751 rolU ribosomal protein L21 (E-Sol, 42.3\%)

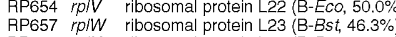

$\begin{array}{lll}\text { RP657 } & \text { rolW } & \text { ribosomal protein L23 (B-Bst, } 46.3 \% \\ \text { RP648 } & \text { rpiX } & \text { ribosomal protein L24 (B-Bst, } 55.9 \%\end{array}$

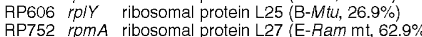

RP099 rpmB ribosomal protein L28 (B-Mge, 43.7\%)

RP651 rpmC ribosomal protein L29 (B-Bst, 39.4\%)

RP100 romE ribosomal protein L31 (B-Mtu, 31.6\%

RP773 rpmF ribosomal protein L32 (B-Hin, 49.1\%

RP879 rom G ribosomal protein L33 (B-Hin, 51.8\%

RP608 rpm/ ribosomal protein L35 (E-Ppu cp, 38.5\%)

RP521 rpsA ribosomal protein S1 (B-Ame, 48.6\%)

RP086 rpsB ribosomal protein S2 (B-Mtu, 41.5\%)

RP653 rpsC ribosomal protein S3 (B-Bst, 54.2\%)

$\begin{array}{lll}\text { RP345 } & \text { rpsD } & \text { ribosomal protein S4 (B-Bsu, 43.2 } \\ \text { RP642 } & \text { rosE } & \text { ribosomal protein S5 }\end{array}$

RP039 rpSF ribosomal protein S6 (B-Hin, 30.2\%)

RP131 rpsG ribosomal protein S7 (E-Ram mt, 45.2\%)

RP645 rpsH ribosomal protein S8 (B-Bsu, 42.0\%)

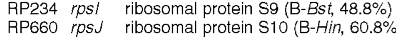

RP636 rpsK ribosomal protein S11 (B-Syn, $53.5 \%$ )

RP130 rosL ribosomal protein S12 (B-Tth, $60.5 \%$ )

AP646 rpsN ribosomal protein S14 (B-Syn, 47.0\%)

RP503 rosO ribosomal protein S15 (B-Bst, $53.4 \%$ )

RP878 rpsP ribosomal protein S16 (B-Hin, 45.1\%)

$\begin{array}{lll}\mathrm{AP} 650 & \mathrm{rpsQ} & \text { ribosomal protein S17 (B-Tth, 61.8\%) } \\ \text { RP040 } & \text { ros R } & \text { ribosomal protein S18 (B-Syn, 50.7\%) }\end{array}$

RPO40
RP655
rpsS ribosomal protein S18 (B-Syn, 50.7\%)

RP615 rpsU ribosomal protein S21 (B-Bbu, $33.3 \%)$

TRANSPORT AND BINDING PROTEINS .

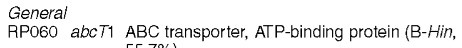

RP508 abcT2 ABC transporter, ATP-binding protein (B-Rme,
RP214 $48.8 \%$ )

214 abcT3 ABC transporter, ATP-binding protein (B-Hin

RP387 $m s b A 1$ ABC transporter, ATP-binding protein (B-Eco,

$26.2 \%)$
RP696 $m s b A 2 \mathrm{ABC}$ transporter, ATP-binding protein (B-ECO,
$28.2 \%)$ $28.2 \%$ )

Amino acids
RP307 atrc1 cationic amino acid transporter (E-Mmu, 29.7\%) RP129 ginP glutamine transport system permease (B-Bsu,

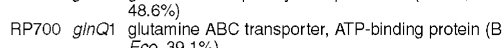

RP868 gin Q2 glutamine ABC transporter, ATP-binding protein (B-

RP176 gitP $\begin{aligned} & \text { Bst, } 51.0 \% \text { ) } \\ & \text { glutamate-aspartate transporter (B-Bca, 35.2\%) }\end{aligned}$

RP483 potE putrescine-ornithine transporter (B-Hin, $26.9 \%$ )
RP369 potG

RP077 proP1 Mpn, $29.2 \%$ )

RP313 proP2 proline/betain transporter (B-ECO, 24.9\%

RP685 proP4 proline/betain transporter (B-ECO, 21.2\%

RP755 proP5 proline/betain transporter (B-ECO, 27.8\%

RP852 proP6 proline/betain transporter (B-ECO, 34.8\%

RP881 prof7 proline/betain transporter (B-Eco, $28.7 \%$ )
RP150 yqiX amino acid ABC transporter (B-Bsu, 32.4\%)

Nucleosides and nucleotides
RP097 $\mathrm{mkl}$ ribonucleotide ABC transporter, ATP-binding protein

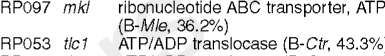

$\begin{array}{ll}\text { RP377 tic2 } & \text { ATP/ADP translocase (B-Ctr, } 35.2 \% \\ \text { RP477 tic3 } & \text { ATP/ADP translocase }(B-C t, 39.6 \%\end{array}$

RP500 tic4 ATP/ADP translocase (B-Ctr, 36.3\%)
RP739 tic5 ATP/ADP translocase (B-Ctr, 34.7\%)

Carbohydrates, organic alcohols and acids
RP054 gipT glycerol-3-phosphate permease (B-Bsu, $37.1 \%$ )

Cations

Cations
RP834 afuC iron ABC transporter, ATP-binding protein (B-Eco,

RP810 kefB $33.9 \%$ )

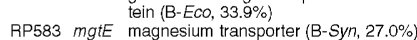

Other aim 1 mitochondrial ABC transporter, ATP-binding protein
RP205 aim

RP794 ccmA (E-Sce, 43.3\%)

RP268 ccmB haem exporter protein B (E-Ram mt, 20.9\%)

P8571 ccmC haem exporter protein C (B-Bja, 43.7\%)

RP630 perM permease PerM homologue (B-Hin, 25.0\%)

RP374 sec7 transport protein Sec7 (B-Hsa, 29.6\%)
RP576 prsA protein export (B-Bsu, 28.9\%)

OTHER CATEGORIES

.52

Adaptations to atypical condition

RP708 himA integration host factor $\alpha$ (B-Eco, 29.5\%)

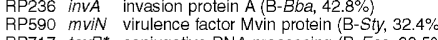

RP717 taxB* conjugative DNA processing (B-ECO, 33.5\%)

RP286 trbG conjugal transfer (B-Rsn, 24.7\%)

RP784 virB4 virulence protein VIRB4 (B-Atu, 20.3\%)

RP287 virB8 virulence protein VIRB8 (B-Atu, 20.4\%)

RP291 virB10 virulence protein VIRB10 (B-Atu, 20.3\%)

RP292 virB11 virulence protein VIRB11 (B-Atu, 29.6\%)
RP293 virD4 virulence protein VIRD4 (B-Atu, 31.3\%)

Drug and analogue sensitivity

RP170 acrD acriflavin resistance protein D (B-Eco, 31.3\%)

RP475 ampG1AMPG protein (B-ECO, 31.4\%)

RP668 ampG2AMPG prt (B-ECO, 26.3\%

RP603 bcr bicyclomycin resistance (B-Eco, 21.7\%)

$\begin{array}{lll}\text { RP698 } & b c / 2 & \text { bicyclomycin resistance (B-Eco, } 18.8 \%) \\ \text { RP243 emra } & \text { multidrug resistance protein A (B- } E c 0,26.9 \%)\end{array}$

RP157 emrB multidrug resistance protein B (B-Hin, 29.3\%)

RP786 terC tellurite resistance protein (B-ECO, $35.3 \%$ )

Colicin-related functions

RP302 tolB colicin tolerance protein (B-Hin, 29.8\%)

RP309 tolQ inner membrane protein (B-Eco, 39.7\%)
RP310 tolR inner membrane protein (B-Pae, 40.1\%)

Uncategorized

RP493 addA adducin alpha subunit (E-Hsa, 32.6\%)
RP199 adx1 adrenodoxin precursor (E-Spo, 57.1\%)

RP714 ank2 ankyrin (E-Hsa, 32.7\%)
RP245 bolA BolA protein (B-Val, 34.2\%)

$\begin{array}{ll}\text { RP245 bolA } & \text { BolA protein (B-Val, 34.2\%) } \\ \text { RP181 ctaQ* } & \text { thermostable carboxypeptidase (B-Pho, 29.1\%) }\end{array}$

RP181 $\mathrm{ctaQ}^{*}$ thermostable carboxypeptidase (B-Pho, 29.1\%)
RP297 cysQ sulphite synthesis pathway protein (B-Eco, 31.2\%)

RP323 cyaY CyaY protein (B-Ech, $31.1 \%$ )
RP118 era GTP binding protein Era (B-Bsu, $33.6 \%)$

RP063 hesB1 HesB protein (B-Ava, 37.0\%)

RP484 hesB2 HesB protein (B- $P$ bo, 40.2\%)

RP485 nifU nitrogen fixation protein (B-Avi, 43.0\%)

RP602 pat1 patatin B1 precursor protein (E-Stu, 22.9\%)

RP317 pkcl protein kinase C inhibitor (B-Abr, 38.6\%)

RP109 ptb phosphate buturyltransferase (B-Cab, $36.4 \%)$
RP594 sco $\mathrm{B}^{*}$ succinyl-CoA:3-ketoacid-CoA transterase subunit B

$\begin{array}{ll}\text { RPO31 sco2 } & \text { (B-Mitu, } 22.0 \% \text { ) } \\ \text { Sco yeast precursor protein (E-Sce, 32.6\%) }\end{array}$

$\begin{array}{lll}\text { RPP87 } & \mathrm{scO2} & \text { Sco yeast precursor protein } \\ \text { RP846 } & \text { sthB } & \text { SthB protein (B-Zmo, } 40.6 \%\end{array}$

RP430 smpB small protein (B-Syn, 46.7\%

RP058 soj SOJ protein (B-Bsu, 50.4\%)

RP486 spl1 tRNA splicing protein (E-Cal, $58.9 \%$ )

AP487 sp/1 tANA splicing protein (E-Cal, 32.3\%)

RP259 spoOJ sporulation protein (B-Bsu, 40.4\%)
RP239 suhB supressor protein (B-Eco, 22.8\%)

RP733 surfy SurF1 protein (E-Hsa, $23.9 \%$ )

HYPOTHETICAL PROTEINS.
Integral membrane proteins 10

NO SIMLARITY

Integral membrane proteins 21 